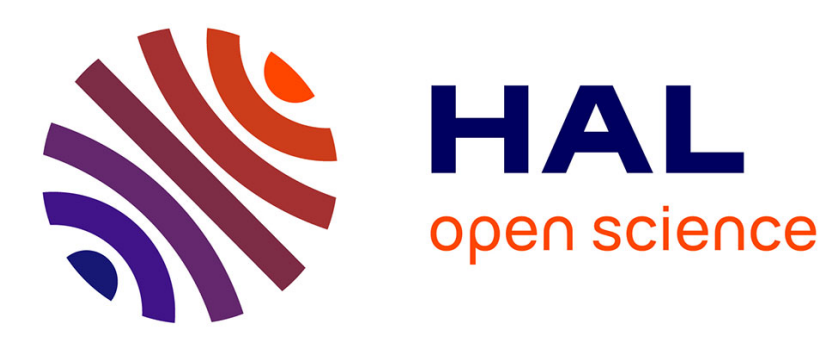

\title{
A two-stage game theoretical approach for interference mitigation in Body-to-Body Networks
}

\author{
Amira Meharouech, Jocelyne Elias, Ahmed Mehaoua
}

\section{To cite this version:}

Amira Meharouech, Jocelyne Elias, Ahmed Mehaoua. A two-stage game theoretical approach for interference mitigation in Body-to-Body Networks. Computer Networks, 2016, 95, pp.15 - 34 . 10.1016/j.comnet.2015.12.001 . hal-01374322

\section{HAL Id: hal-01374322 \\ https://hal.science/hal-01374322}

Submitted on 30 Sep 2016

HAL is a multi-disciplinary open access archive for the deposit and dissemination of scientific research documents, whether they are published or not. The documents may come from teaching and research institutions in France or abroad, or from public or private research centers.
L'archive ouverte pluridisciplinaire HAL, est destinée au dépôt et à la diffusion de documents scientifiques de niveau recherche, publiés ou non, émanant des établissements d'enseignement et de recherche français ou étrangers, des laboratoires publics ou privés.

$$
\text { Copyright }
$$




\title{
A Two-Stage Game Theoretical Approach for Interference Mitigation in Body-to-Body Networks
}

\author{
Amira Meharouech ${ }^{\mathrm{a}}$, Jocelyne Elias ${ }^{\mathrm{a}, *}$, Ahmed Mehaoua $^{\mathrm{a}}$ \\ ${ }^{a}$ LIPADE Laboratory, Université Paris Descartes - Sorbonne Paris Cité, 75006 Paris, France.
}

\begin{abstract}
In this paper, we identify and exploit opportunities for cooperation between a group of mobile Wireless Body Area Networks (WBANs), forming a Body-to-Body Network (BBN), through inter-body interference detection and subsequent mitigation. Thus, we consider a dynamic system composed of several BBNs and we analyze the joint mutual and cross-technology interference problem due to the utilization of a limited number of channels by different transmission technologies (i.e., ZigBee and WiFi) sharing the same radio spectrum. To this end, we propose a game theoretical approach to address the problem of Socially-aware Interference Mitigation (SIM) in BBNs, where WBANs are "social" and interact with each other. Our approach considers a two-stage channel allocation scheme: a BBN-stage for inter-WBANs' communications and a WBAN-stage for intra-WBAN communications. We demonstrate that the proposed BBN-stage and WBAN-stage games admit exact potential functions, and we develop a Best-Response (BR-SIM) algorithm that converges to Nash equilibrium points. A second algorithm, named Sub-Optimal Randomized Trials (SORT-SIM), is then proposed and compared to BR-SIM in terms of efficiency and computation time. We further compare the
\end{abstract} BR-SIM and SORT-SIM algorithms to two power control algorithms in terms of signal-to-interference ratio and aggregate interference, and show that they outperform the power control schemes in several cases. Numerical results, obtained in several realistic mobile scenarios, show that the proposed schemes are indeed efficient in optimizing the channel allocation in medium-to-large-scale BBNs.

Keywords: Body-to-Body Networks, Interference Mitigation, Cross-Technology

Interference, Channel Allocation, Game Theory, Nash Equilibrium. 


\section{Introduction}

Body-to-Body Networks (BBNs) have recently emerged as a promising solution for monitoring the people behavior and their interactions with the surrounding environment [2].

The BBN consists of several WBANs, which in turn are composed of sensor nodes that are usually placed in the clothes, on the body or under the skin [3]. These sensors collect information about the person and send it to the sink (i.e., a Mobile Terminal (MT) or a PDA), in order to be processed or relayed to other networks (Fig.1) .

BBNs are widely adopted in several mission-critical scenarios: (i) rescue teams in a disaster area, (ii) groups of soldiers on the battlefield [4], and (iii) patients in a healthcare center, whose Wireless Body Area Networks (WBANs) interact with each other. Yet, the $\mathrm{BBN}$ can be implemented in both medical and non-medical applications. Indeed, BBNs represent the novel trend for future, ubiquitous healthcare systems, in which the remote monitoring of patients carrying bodyworn sensors and relaying each others physiological data up to the medical center, could greatly reduce the current strain on health budgets and make the Government's vision of ubiquitous healthcare for distant patients a reality. For example, when a patient is at home or far from the medical center, and feels a sudden trouble, she will be able to broadcast a distress call and bring out an urgent human assistance from his neighborhood. Hence, the sensors could be embedded into mobile handsets, portable electronic devices, cars, and clothing. Thanks to low-power body-tobody networks, people would no longer need to be in the range of a cellular tower to make a call or transmit data. Fig.2 sorts the different BBN applications into medical and non-medical classes, and lists the new intended applications by the deployment of BBN networks. Due to the scarce wireless resources, many existing wireless technologies, like IEEE 802.11 (WiFi), IEEE 802.15.1 (Bluetooth) and IEEE 802.15.4 (ZigBee), are forced to share the same unlicensed 2.4 GHz Industrial, Scientific and Medical (ISM) band. Hence, mutual as well as cross-technology interference may occur between these

\footnotetext{
Very preliminary results of this work have been presented in [1].

* Corresponding author

Email addresses: amira.meharoueche@etu.parisdescartes.fr (Amira Meharouech), jocelyne.elias@parisdescartes.fr (Jocelyne Elias), ahmed.mehaoua@parisdescartes.fr (Ahmed Mehaoua)
} 


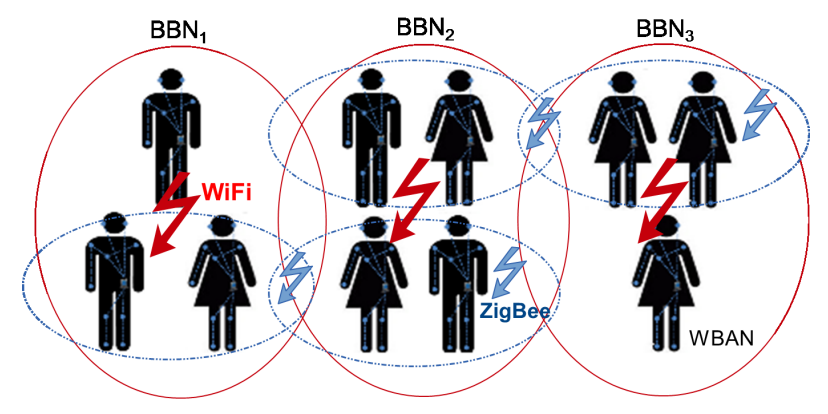

Figure 1: Three-BBN interfering scenario: each BBN is composed of several WBANs which use different transmission technologies (i.e., ZigBee and $\mathrm{WiFi}$ ) sharing the same radio spectrum.

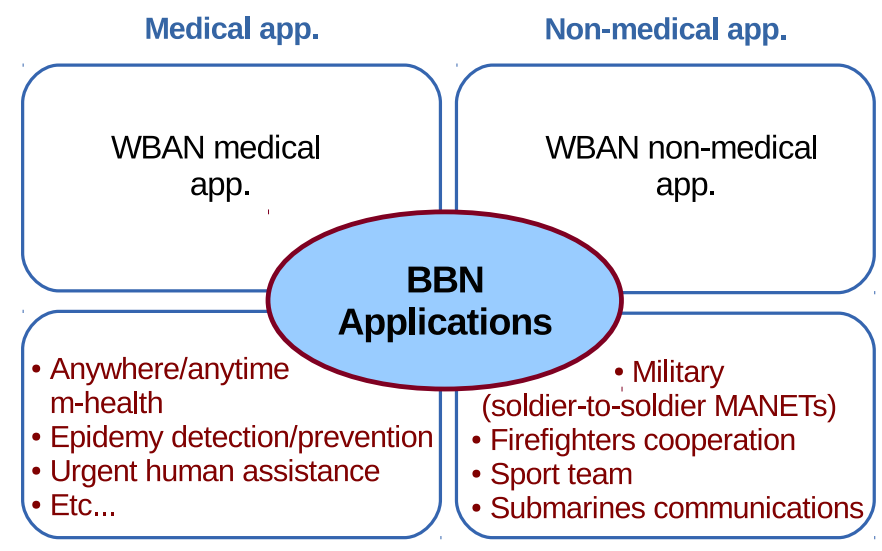

Figure 2: Application area extensions from WBAN to BBN

technologies.

Indeed, the interference issue is already handled by the Bluetooth Low Energy (BLE) standard [5], which defines three channels as advertising channels, used for device discovery and connection establishment, and have been assigned center frequencies that minimize overlapping with IEEE 802.11 channels 1, 6 and 11, which are commonly used in several countries. Then, an adaptive frequency hopping mechanism is used on top of the 37 data channels in order to face interference and wireless propagation issues, such as fading and multipath. This mechanism selects one of the 37 available data channels for 
communication during a given time interval, so as to avoid interference with neighboring wireless links. Furthermore, a number of previous works enhanced the existing frequency hopping mechanism and implemented further schemes, such as the OverLap Avoidance (OLA) proposed in [6].

Coexistence and interference mitigation between WBANs are also considered by the IEEE 802.15.6 standard. Three mechanisms are defined: beacon shifting, channel hopping and active superframe interleaving [7]. Yet, our choice for ZigBee aims at effectively and theoretically tackling the cross-technology interference problem between WiFi (802.11) and ZigBee (802.15.4) technologies.

Since WiFi transmission power can be 10 to 100 times higher than that of ZigBee, ZigBee communication links can suffer significant performance degradation in terms of data reliability and throughput. In addition to the previously mentioned challenging issues, the mobility of WBANs in their surrounding environment and their interactions with each other make the interference mitigation in body-to-body networks a very interesting and mandatory problem to address. This is indeed the main focus of our work.

In this paper we consider a multi-BBN scenario (an example scenario, with 3 BBNs, is illustrated in Fig.1), composed of a set of WBANs that share the same ISM band, and we address the mutual and cross-technology interference mitigation problem introducing a new game theoretical approach. The proposed approach consists of two nested games. The first game aims to allocate WiFi channels for inter-WBANs' wireless communications. Specifically, special players (which are called "delegates" or "leaders") decide the allocation of the needed WiFi channels for themselves and the underlying subnetworks by maximizing an utility function, which is a function of mutual and crosstechnology Signal-to-Interference Ratio (SIR) metric. The second proposed game is a WBAN-stage SIM game that allows players (or WBANs) to choose the needed ZigBee channels for intra-WBAN communications, taking into account the allocations performed by the BBN-stage SIM game.

The main contributions of our work are the following:

- We propose a novel game theoretical approach for mutual and cross-technology interference mitigation in BBNs.

- We provide a detailed expression of the Signal-to-Interference Ratio to define play- 
ers' payoff functions, capturing all main interference components, namely the cochannel, the mutual, and the cross-technology interference.

- We demonstrate that our games admit at least one pure strategy Nash Equilibrium (NE) since they are exactly potential, and we develop best response algorithms (BR-SIM) to compute the channel allocations, which converge fast to NE solutions.

- We propose a second algorithm, called Sub-Optimal Randomized Trials (SORTSIM), that trades-off between efficient channel allocation process and short computation time, and guarantees a sub-optimal solution to the SIM problem.

- We perform a thorough performance analysis of the BBN- and WBANstage SIM games under different system parameters, and compare the two proposed algorithms, i.e., BR-SIM and SORT-SIM to a distributed power control and a relay-assisted power control algorithm. Numerical results show that the proposed schemes are indeed efficient in optimizing the channel allocations in medium-to-large-scale realistic mobile BBN scenarios.

The paper is structured as follows: Section 2 discusses related work. Section 3 presents the BBN system model, including the communication and the interference model. Section 4 details the two-stage Socially-aware Interference Mitigation (SIM) game theoretical approach. Section 5 presents the best-response algorithm (BR-SIM), while Section 6 handles the sub-optimal solution (SORT-SIM) for the SIM problem. Section 7 analyzes numerical results for the proposed solutions in several BBN scenarios. Finally, Section 8 concludes this paper.

\section{Related Work}

In this section, we discuss the most relevant works that deal with the problem of interference mitigation between different technologies (i.e., Bluetooth, ZigBee, WiFi) that share the same frequency spectrum.

Whilst a number of previous interference-aware studies have been based upon power considerations $[8,9]$, others have chosen different alternatives $[10,11]$ to deal with this 
substantial problem which is challenging in WBAN design, and raising even more with the emergence of BBNs.

In [8] the authors propose a distributed power control algorithm which converges to the Nash Equilibrium, representing the best tradeoff between energy and network utility. No transmissions are envisaged among WBANs in [8]; a transmission is either from a WBAN node to its gateway or vice versa, neither access technology assumption is made, it is rather assumed that only mutual interference could happen. However, in a BBN context where WBANs communicate with each other, it is mandatory to consider transmissions among WBANs' gateways and thus investigate cross-interference scenarios where different wireless technologies could be used for intra-WBAN and inter-WBANs transmissions scenarios.

While most power control models provide interference-aware schemes over power adaptation, authors of [9] optimized a transmission scheme given a constant power. They formulated an interference-aware channel access game to deal with the competitive channel usage by different wireless technologies sharing the ISM band, in both static and dynamic scenarios. Using Game Theory, authors of [9] stated that a decentralized approach is resilient to users' deviation and ensures the robustness of the network, compared to a centralized approach where the system cannot be easily protected from a selfish deviation to increase, unilaterally, one's throughput. Alike our BBN model, this game considers nodes concurrently transmitting in nearby clusters, incorporating the Signal-to-Interference-plus-Noise Ratio (SINR) model as wireless communication metric. Nonetheless, the game focuses on the channel access problem under inter-cluster interference from nearby APs using the same wireless technology, while the key advantage of our work is to consider both mutual and cross-technology channel interference problems.

Game theory is applied in such distributed problems, such as in [10], where the multichannel usage problem in Wireless Sensor and Actuator Networks (WSANs) is modeled as a channel allocation game with the total interference of the whole network as the social objective to minimize. In WSANs, communication and control are highly integrated, even though each node (a sensor, actuator or control unit) is equipped exclusively with one simple half-duplex radio transceiver. However, the major difference with our network model is that BBNs are randomly distributed networks where underlying WBANs are 
mobile and equipped with two radio antennas to ensure on-body and off-body communications. Yet, WBANs may randomly overlap with each other, which makes BBN a highly dynamic system over time and space, compared to WSNs, apart from the human body environment challenge related to WBANs. Yet, further constraints are to be considered to design an effective channel allocation scheme for BBNs.

On the other hand, the main idea in [11] is that using only power control to combat this interference might not be efficient; it could even lead to situations with higher levels of interference in the system. Therefore, the work in [11] proposes several interference mitigation schemes such as adaptive modulation as well as adaptive data rate and adaptive duty cycle. Interference Mitigation Factor is introduced as a metric to quantify the effectiveness of the proposed schemes. Based on SINR measurements, these schemes are likely suitable for small-scale WBANs where SINR is function of the transmit power, such as in [8] which uses the SINR metric as a utility function to model the interference problem between neighboring WBANs considering a power control game. In fact, in [8] the network topology is static and no actual communications among WBANs are considered. However, in [12], an experimental study proved the importance of the impact of human body shadowing in off-body communications. Yet, for relatively complex BBNs, SINR is also highly dependent on outdoor conditions and human body effects, and the aforementioned schemes would no longer be efficient, or they should be extended taking into account additional physiological, physical, and environmental parameters. Particularly, in dynamic scenarios, when the SINR is varying due to the fast topology changes with neighboring WBANs movements, relying only on the transmit power in order to keep the desired link quality might not be effective. Indeed, in a BBN scenario with high transmit power from other coexisting wireless networks/WBANs, the interference is significant and the desired link quality cannot be achieved unless considering the surrounding conditions (interference) and the wireless channel characteristics in terms of shadowing, fading, etc., which can be incorporated into the channel gain parameters of the SINR.

Besides, several works investigated the interference mitigation problem with detailed specifications of wireless technologies, especially WiFi, ZigBee, and Bluetooth, which are very popular in the WBAN industry. For example, authors of [13] proposed an approach 
that accurately characterizes the white space in WiFi traffic and develop a ZigBee frame control protocol called WISE, which can predict the length of white space in WiFi traffic and achieve desired trade-offs between link throughput and delivery ratio. The empirical study of ZigBee and WiFi coexistence provided by [13] is useful to understand and model the cross-technology problem. Nevertheless, the WiFi-WiFi and ZigBee-ZigBee mutual interference problems still need to be carefully investigated, especially when coupled with mobility, topology changes and other features related to the complexity of BBN networks, which require more intelligent functions at the WBAN coordinator's (MT) level, in order to ensure an effective channel allocation scheme for BBNs. Further studies [14, 15, 16] have dealt with the solutions that enable ZigBee links to achieve guaranteed performance in the presence of heavy WiFi interference, but almost all of them propose approaches that assume having already established the ZigBee and WiFi links, and try to implement mechanisms to mitigate the interference between them.

In [17], the authors provided an interesting study that explores the possibility of exploiting Partially Overlapped Channels (POCs) by introducing a game theoretic distributed Channel Assignment (CA) algorithm in Wireless Mesh Networks (WMNs). The proposed CA algorithm aims at increasing the number of simultaneous transmissions in the network while avoiding signal interference among multi-radio nodes. A Cooperative Channel Assignment Game (CoCAG) is implemented, where information is exchanged with neighboring nodes. In fact, by considering neighboring information, nodes can track the instantaneous neighbors' strategies when assigning channels to themselves, which can help in guaranteeing a fair sharing of the frequency band. The major contribution of [17] is that it addresses four different types of interference and their influence on the network capacity: Co-channel Interference, Orthogonal Channels, Adjacent Channel Interference and Self Interference. Nonetheless, one key feature of the WMN is the backbone network composed of Mesh Routers that are usually static and have no constraints on energy consumption, which is not the case for WBANs. Moreover, only IEEE 802.11g was used as wireless technology in [17], and as a consequence no cross-technology scenarios were considered.

Again, in order to cope with the interference issue in WBANs, authors of [18] implemented an intelligent power control game which allows WBANs to improve their 
performance by learning from history. The proposed power controller implements a genetic algorithm $(\mathrm{GA})$ which enables WBANs to learn from experience and select their power strategies in a distributed manner with no inter-node negotiation or cooperation. Authors state that less inter-node interactions are more attractive for WBANs due to their low overhead and superior scalability. However, such assumption barely adapts to our network model, due to the ever changing topology, the highly dynamic outdoor environment, and the continuously joining and leaving WBANs typical of a BBN scenario.

In [19], we addressed the interference mitigation problem for BBNs considering a centralized approach and we formulated it as an optimization problem. To solve efficiently the problem even for large-scale network scenarios, two heuristic solutions were developed, namely, a customized randomized rounding approach and a tabu search scheme. Our work differs from [19] in two main aspects: (1) we formulate the problem of mutual and cross-technology interference mitigation, considering the Signal-to-InterferenceRatio (SIR) and a noise component related to physical conditions and human body effects, and we therefore allocate WiFi/ZigBee wireless channels to communication links optimizing the SIR ratio, while in [19] the interference was only quantified by the binary decision variables; (2) we address the interference mitigation problem using a distributed approach, with concepts and mathematical tools from Game Theory, while this problem was tackled in [19] in a completely centralized way.

Yet, to the best of our knowledge, this paper is the first to propose a game theoretical approach for an interference-aware channel allocation in BBNs. In our model, multiple WBANs could interact among each other within a BBN, as well as with other coexisting networks/BBNs, involving different access technologies (WiFi, ZigBee, Bluetooth..); this can lead to unavoidable heavy interference environment.

\section{System Models}

In this section, we present the system models, including the network model and the interference model, arising in body-to-body networks.

\subsection{Network Model}

We consider a BBN scenario composed of a set $\mathcal{N}$ of WBANs, which are located in the same geographical area (i.e., a medical center, a rest home or a care home), and 
share the same unlicensed 2.4 GHz ISM band. Let $\mathcal{C}^{w}$ and $\mathcal{C}^{z}$ denote, respectively, the set of WiFi and ZigBee channels in this band.

Each WBAN is equipped with a wearable Mobile Terminal $(\mathrm{MT})^{1}$, that uses both the 802.15.4 protocol (i.e., ZigBee) to communicate with the sensor nodes within its WBAN, and the IEEE 802.11 wireless standard (i.e., WiFi) to create a backhaul infrastructure for inter-WBANs' communications.

Since we are assuming that WBANs can move and interact with their surrounding environment, we find ourselves in a quite dynamic BBN scenario, and therefore, we decide to divide the operating time of the whole system into a set $T$ of consecutive epochs, and during each epoch $t \in T$ we suppose that the network topology and environment conditions do not change.

The set $\mathcal{L}^{w}(t)$ represents all WiFi unidirectional links established by mobile terminals during the epoch $t \in T ; \mathcal{L}^{w}(t)$ may vary between two consecutive epochs due to WBANs' mobility. On the contrary, the set $\mathcal{L}^{z}$, which represents the ZigBee unidirectional links used for intra-WBAN communication among the sensors, does not change with time, and for this reason, we omitted the parameter $t$ from this set.

Recent works dealing with interference mitigation have considered the binary model to represent overlapping between channels $[13,14,15,16,19]$; i.e. a node is either interfered or not, however our idea in this work is to quantify the interference between partially overlapped channels. In [20], the authors model the overlapping among different WiFi channels defining a symmetric channel overlapping matrix $W$, whose element $w_{m n}$ quantifies the degree of interference between channels $m$ and $n$, and is given as follows:

$$
w_{m n}=\frac{\int_{-\infty}^{+\infty} F_{m}(w) F_{n}(w) d w}{\int_{-\infty}^{+\infty} F_{m}^{2}(w) d w},
$$

where $F_{m}(w)$ and $F_{n}(w)$ denote the power spectral density (PSD) functions of the bandpass filters for channels $m$ and $n$, respectively, which can be obtained from the channels' frequency responses. Yet, we need to know which channel filter is being used. As in [20], we assume the use of raised cosine filters, whose principle is explained in [21]. Fig.3 shows how the PSD function of the IEEE $802.11 \mathrm{~b}$ depends on the roll-off factor $\delta$, which

${ }^{1}$ The $W B A N$ and his corresponding Mobile Terminal will be used as synonyms throughout the paper. 
is a key parameter of the raised cosine filter, when it is equal to 1 and 0.25 , respectively. Hence, [21] gives a simplified expression of the $W$ matrix :

$$
w_{m n}=w_{n m}=\frac{A_{o}}{A_{o}+A_{n o}}
$$

where $A_{o}$ and $A_{n o}$ are the overlapping and non-overlapping areas between the power spectral density (PSD) of channels $m$ and $n$, respectively. With expression (2), the $W$ matrix can be computed off-line and used as a constant matrix in the BR-SIM and SORT-SIM algorithms.

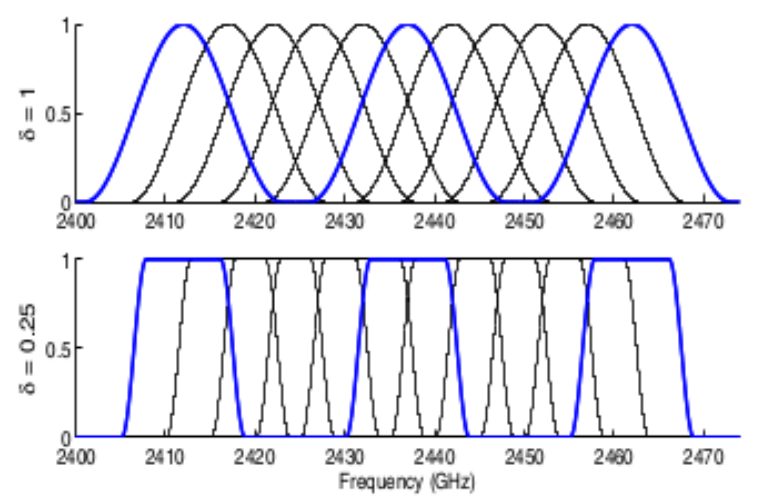

Figure 3: The $802.11 \mathrm{~b}$ frequency responses with the raised cosine filter [21].

Since different wireless technologies use different signal modulations and access mechanisms, authors of [22] performed an extensive set of experiments to measure the partial overlap of the IEEE 802.11b standard, using different physical layer modulation methods. First, they considered 1 and 2 Mbps data-rates for the physical layer, using the Binary Phase Shift Keying (BPSK) modulation, to measure the channel overlap. Then, they reported results using the Complementary Code Keying (CCK) modulation, with a data-rate of $11 \mathrm{Mbps}$. It was concluded that partially overlapped channels can provide much greater spatial re-use if used carefully, depending on the physical separation and/or the channel separation between neighboring links, whatever the modulation scheme in 
use.

In this work, we model the channel overlapping problem analytically by studying its impact on the signal-to-interference ratio. Yet, although the channel overlapping matrix $W$ has been defined to model the partially-overlapped channels for the $802.11 \mathrm{~b}$ protocol, it does not depend, actually, on the technology in use, since the expression could involve the PSD functions of any frequency responses, provided that the frequency band presents overlapping behaviors, which is actually not the case for ZigBee and BLE, since both frequency bands present orthogonal wireless channels.

To summarize, our network model will focus on the following relevant elements:

- Every single WBAN's MT, equipped with one WiFi antenna and one ZigBee antenna, should dispose of non overlapping WiFi and ZigBee channels.

- No interference is present within a WBAN; we assume a TDMA-based medium access control implemented in each WBAN to deal with collisions. Note in addition that there is no interference between adjacent ZigBee channels since there is no overlapping.

- The interference between overlapping WiFi and ZigBee channels is represented by the matrix $A$, of size $\left|\mathcal{C}^{w}\right| \times\left|\mathcal{C}^{z}\right|$, whose element $a_{c_{1} c_{2}}$ is a binary value: $a_{c_{1} c_{2}}=1$ if WiFi channel $c_{1}$ overlaps with ZigBee channel $c_{2}$ (0 otherwise).

- As in [20], the degree of interference between overlapping WiFi channels is represented by the matrix $W$, of size $\left|\mathcal{C}^{w}\right| \times\left|\mathcal{C}^{w}\right|$, whose element $w_{c_{1} c_{2}} \in[0,1]$ is a fractional value, defined by the expression in Equation (1).

- To preserve the network connectivity within the BBN, we assume that all WBANs WiFi interfaces are tuned on the same channel. Therefore, we use the $\left|\mathcal{L}^{w}\right| \times\left|\mathcal{L}^{w}\right|$ matrix $B(t)$, whose element $b_{i j}$ is a binary value: $b_{i j}=1$ if WiFi links $i$ and $j$ belong to the same BBN at time epoch $t \in \mathcal{T}$ (0 otherwise).

- Finally, WBANs use a higher transmission power on the inter-WBAN channel than on the channel used for intra-WBAN communications (i.e. $p^{w}>>p^{z}$ ). In particular, data transmissions within ZigBee networks can completely starve due to WiFi communications, which use 10 to 100 times higher transmission power [19]. 
In order to minimize the total interference within BBNs involving several wireless technologies, it is advantageous to observe every interference component separately, thus we can specify two-kind interference scenarios:

- The Mutual interference:

- WiFi-WiFi interference at the MT receiver, that occurs while receiving collected data from a nearby WBAN of the same BBN and interfering with adjacent BBNs' WiFi links. Such component includes as well the co-channel interference.

- ZigBee-ZigBee interference at the MT receiver, that happens when a ZigBee link of a WBAN interferes with a ZigBee link of another WBAN belonging to the same or to a different BBN, when they are allocated the same channel.

- The Cross-Technology interference: WiFi-ZigBee, among adjacent WBANs, where each WBAN (MT) is communicating with other WBANs over a WiFi link and is susceptible to interference from nearby ZigBee links, and vice versa.

The Interference issue and the SIR metric are tightly related. Thus, in this paper, we would focus on the interference metric (SIR) expressed in decibel format by:

$$
\operatorname{SIR}_{i}(t)(d B)=10 \log \left(\frac{g_{i i}(t) p^{i}}{\sum_{j \neq i} g_{i j}(t) p_{j}}\right),
$$

where $p^{i}$ is the transmission power of transmitter $i, g_{i j}(t)$ is the link gain from transmitter $j$ to receiver $i$ at time epoch $t$. Since WBANs can move in their surrounding environment, the links' gains $g_{i j}(t)$ vary over time, and the SIR in turn has been further expressed as a function of time $t$.

The gain parameters are calculated taking into account the average channel gain evaluated at the reference distance $d_{0}=1 \mathrm{~m}$ and with a path loss exponent $n(\alpha)$, according to the following formula [23]:

$$
\left.g_{i j}(t)\right|_{d B}=\left.G\left(d_{0}, \alpha\right)\right|_{d B}-10 \times n(\alpha) \times \log _{10}\left(d / d_{0}\right), \quad \forall i, j \in \mathcal{L}^{w}(t) \cup \mathcal{L}^{z}
$$

Specifically, the average channel gain $G\left(d_{0}, \alpha\right)$, between WBANs' MTs (Tx Right Hip, Rx Right Hip), significantly decreases from $-37.88 d B$ to $-66.33 d B$ when switching 
from LOS to NLOS conditions, which ensures that our BBN scenarios are consistent with a realistic human body environment.

\subsection{Interference Model}

The interference model defines the set of links that can interfere with any given link in the network [24]. There have been various interference models proposed in the literature; the common concept is that two communication links $i=\left(T_{i}, R_{i}\right)$ and $j=\left(T_{j}, R_{j}\right)$ are interfering if and only if either $T_{i}$ or $R_{i}$ lies within the interference range of $T_{j}$ or $R_{j}$, where $T_{i}, T_{j}$ and $R_{i}, R_{j}$ designate the transmitter and receiver interfaces of links $i$ and $j$, respectively.

If modeling the interference characteristics in sensor networks is challenging, it is more so for BBNs, because RF characteristics of nodes and environments are neither known a priori nor computable due to their stochastic, rapidly changing characteristics [25]. Any routing protocol working in high interference environment is incapable of dealing with radio channels suffering from high interference ratios. Thus, sharing channels appropriately according to the interference profiles is mandatory and prior for BBN networks design.

Interference range is the range within which nodes in receive mode will be interfered with an unrelated transmitter and thus suffer from packet loss [26]. For simplicity, ranges are generally assumed concentric which is not necessarily given in physical networks. In [26], the interference range was defined based on SIR, where authors assume a transmission scenario with transmitter-receiver distance as $d$ meters and at the same time, an interfering node $r$ meters away from the receiver, starts another transmission. The received signal is assumed to be successful if it is above a SIR threshold $\left(S I R_{t h}\right)$.

Conflict Graph Given an interference model, the set of pairs of communication links that interfere with each other, assuming mutual and cross-interference in our model, can be represented using a conflict graph. As done in $[19,27]$, we depict a conflict graph to model the mutual and cross-technology interfering wireless links. We adopt this representation because it will help us in defining the set of neighbors in next sections for our Socially-aware Interference Mitigation game. Therefore, the cross-technology conflict graph $G_{c}\left(V_{c}(t), E_{c}(t)\right)$ is defined as follows: 
- $V_{c}(t)$ : set of vertices corresponding to WiFi and ZigBee communication links in the network, $V_{c}(t)=\mathcal{L}^{w}(t) \cup \mathcal{L}^{z}$.

- $E_{c}(t)$ : set of edges corresponding to the interference relationship among pairs of links. Fig.4 depicts the cross-technology conflict graph of the three BBN-scenario illustrated in Fig.1. Solid lines represent conflict edges between two vertices using the same radio technology, i.e. $\left(e_{1}, e_{2}\right) \in E_{c}(t)$ is a conflict edge if and only if $e_{1}, e_{2} \in \mathcal{L}^{w}(t)$ or $e_{1}, e_{2} \in \mathcal{L}^{z}$, and they are interfering with each other. Whereas dashed lines correspond to cross-conflict edges between two vertices using different radio technologies.

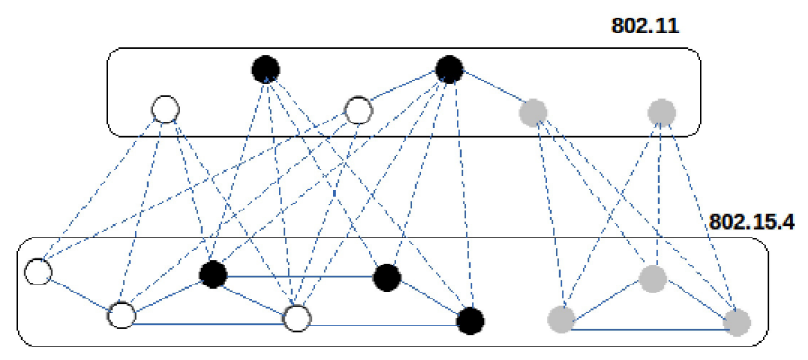

Figure 4: Cross-technology Conflict Graph of the scenario illustrated in Fig.1

Our goal is to minimize the overall network interference. To give an example, let us consider the scenario of Fig.1. Each BBN has different interference ranges with its neighboring BBNs. Assuming that only three WiFi orthogonal channels from the 2.4 $\mathrm{GHz}$ band are available (1, 6, and 11), one trivial solution would be to assign channels 1 , 6 and 11 to BBN1, BBN2 and BBN3, respectively. In this case there would be no interference. Let us assume now that only two WiFi orthogonal channels 1 and 6 are available, in addition to channel 2 overlapping with channel 1. Thus, channels 1, 6 and 2 would be assigned to BBN1, BBN2 and BBN3, respectively. Since BBN1 and BBN3 have disjoint interference ranges, they can use overlapping channels with minimal risk of interference. In practice, the system is more complex, with many more BBNs, and/or more overlapping interference ranges, involving several wireless technologies. Therefore a general approach should be investigated for an appropriate wireless resource sharing according to the interference profiles. Likewise, in such heterogeneous wireless systems, a two-stage channel allocation scheme is needed; a BBN level game for WiFi channel 
allocation step, then a WBAN level game for ZigBee channel allocation, taking into account the cross-technology features at each stage.

\section{Two-stage Socially-aware Interference Mitigation Game (SIM)}

In this section, we first define the basic notation and parameters used hereafter, and then we describe in detail the proposed socially-aware interference mitigation game theoretical approach.

The lack of a centralized control and prioritization of access to the radio spectrum, in addition to the restricted knowledge of network information, motivate us to employ local interactions for the WiFi and ZigBee level games, in which players consider their own payoffs as well as those of their neighbors, so as to optimize their strategies while relying on their surrounding network information. Besides, at the BBN-stage game, each group of interacting WBANs (i.e., each sub-BBN ${ }^{2}$ ) is represented by a special player (a delegate or a leader of the group) who decides which WiFi channel to choose. Indeed, to ensure network connectivity all WBANs within the same sub-BBN should be tuned to the same WiFi channel, and we consider this special player that acts on behalf of the entire sub-BBN. To this end, we consider in this work a two-stage socially-aware interference mitigation scheme:

(i) At a first stage, each BBN takes a decision on the WiFi channel that should be assigned to his WiFi transmission links, ensuring minimal interference with his surrounding environment, through a local interaction game with his neighboring BBNs.

(ii) Then, at the second stage, given the WiFi channel assignment for each BBN, a local interaction game takes place among the WBANs belonging to the same BBN. After playing this game, each WBAN (more precisely, each MT) will be assigned a ZigBee channel to his ZigBee radio interface, and such assignment guarantees the minimal interference of the WBAN with his neighboring WBANs.

The overall operations for the time epoch $t \in T$ are represented by the SIM flow chart given in Fig.5. In this channel assignment game, the players are the set of links

\footnotetext{
${ }^{2}$ The sub-BBN notation is introduced in order to allow different groups of WBANs, belonging to the same BBN, to communicate on different non-overlapping WiFi channels. However, when all WBANs (of the same BBN) want to communicate with each other, then the sub-BBNs coincide with their corresponding BBN.
} 
$\mathcal{L}(t)=\mathcal{L}^{w}(t) \cup \mathcal{L}^{z}$ associated with the set $\mathcal{N}=\{1, \ldots, n\}$ of WBANs occupying either the hospital or a care home for old people, and distributed over a set of coexisting BBNs. Each player is represented by a couple of links $(l, h)$, such that $l \in \mathcal{L}^{w}(t)$ and $h \in \mathcal{L}^{z}$ are a WiFi and a ZigBee link corresponding to a given WBAN $i \in \mathcal{N}$ assimilated to its MT. At time epoch $t \in T$, each player chooses a couple of strategies $\left(s^{l}(t), s^{h}(t)\right) \subset S(t)$, such as $s^{l}(t)$ is the strategy to allocate a WiFi channel $c_{1} \in \mathcal{C}^{w}$ to the WiFi link $l \in \mathcal{L}^{w}(t)$ at time epoch $t \in \mathcal{T}$, denoted by $x_{c_{1}}^{l}$, and $s^{h}(t)$ is the strategy to allocate a ZigBee channel $c_{2} \in \mathcal{C}^{z}$ to the ZigBee link $h \in \mathcal{L}^{z}$, denoted by $y_{c_{2}}^{h} . S(t)$ is obviously the set of the total channel allocation strategies of all players of the BBN scenario. To summarize, the WiFi and ZigBee channel assignment variables are :

$$
\begin{aligned}
& x_{c_{1}}^{l}= \begin{cases}1, & \text { if WiFi channel } c_{1} \text { is assigned to } \\
& \text { the communication link } l \\
0, & \text { otherwise }\end{cases} \\
& y_{c_{2}}^{h}= \begin{cases}1, & \text { if ZigBee channel } c_{2} \text { is assigned to } \\
& \text { the communication link } h \\
0, & \text { otherwise }\end{cases}
\end{aligned}
$$

Hence, hereafter, we first begin with presenting the first-stage game, to choose a WiFi channel assignment for each MT, and then we describe in detail the second-stage game, where each MT is further assigned a ZigBee channel.

\subsection{BBN-stage SIM Game}

In order to assign a single WiFi channel to each sub-BBN, we opt for a BBN-stage SIM game so that each set of communicating WBANs, forming a sub-BBN, are represented by a specific WiFi link. The representative WiFi link is situated in the center of the sub-BBN and plays the role of the delegate, and the other WBANs belonging to the same sub-BBN will be allocated the same WiFi channel (Fig.6). Our choice of the representative WiFi link is similar to the one made by Govindasamy et al. in [28]. In fact, the work in [28] presents a technique to find the spectral efficiency of an interferencelimited representative link with an arbitrary distribution of interference powers, within an ad hoc network with randomly distributed multi-antenna links. This model considers a circular network where the representative receiver is assumed to be at the origin of the circle, and the interferers are links with other receivers whose locations do not impact the 


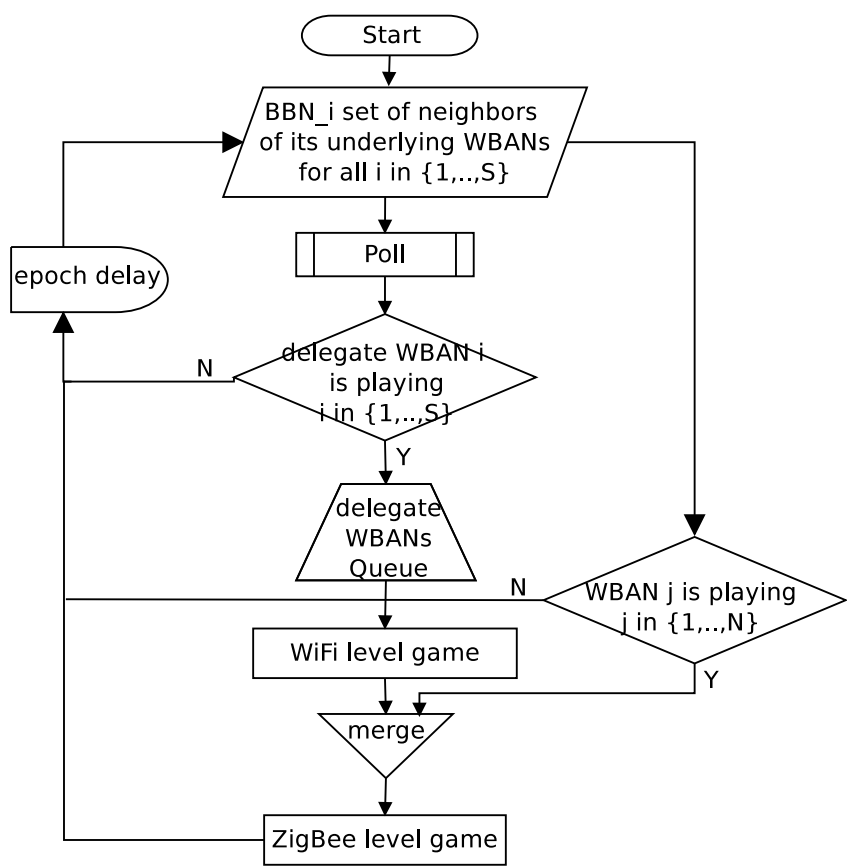

Figure 5: Flowchart of the two-stage SIM Game: (1) Creation of Sub-BBNs and election of the delegates, (2) WiFi level game: Allocation of WiFi channels to the set of WiFi links (represented by their delegates), and (3) ZigBee level game: Allocation of ZigBee channels to ZigBee links of WBANs.

representative link. Of course, there exist a variety of different mechanisms/solutions to select the more appropriate delegate/representative link in the network. However, this issue is not the main concern of this paper and deserves a deep study.

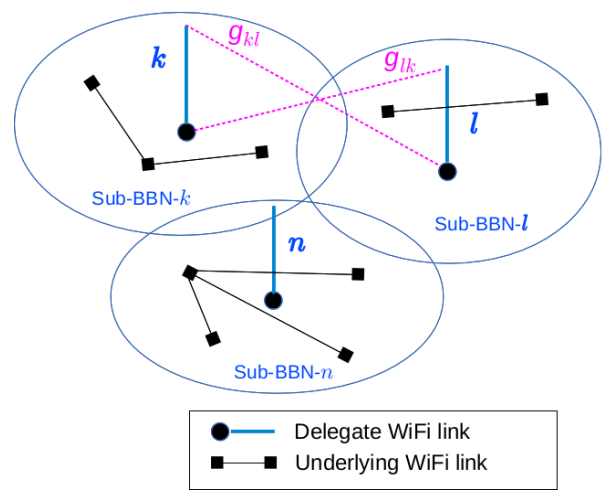

Figure 6: Delegate and underlying WBANs' WiFi links

We build the cross-technology conflict graph and we assume that each WBAN has 
information only about his sub-BBN underlying WBANs, through the exchange of polling messages. Thus, we can identify for each WBAN, the set of interfering neighbors at time epoch $t \in T$ (i.e., the set of edges between a link of such WBAN and transmission links of the others). Let $W_{l}$ denote the set of links interfering with WiFi link $l$ :

$W_{l}(t)=\left\{k \in L^{w}(t):(l, k) \subset E_{c}(t)\right\} \cup\left\{j \in L^{z}:(l, j) \subset E_{c}(t)\right\}$

Thereby, we can define the BBN-stage game $\left(\mathcal{G}_{1}\right)$ as follows:

- Players: the set of BBNs represented by their delegates, such as a delegate player per sub-BBN. For the BBN-stage, the player is assimilated to its WiFi link $l$.

- Strategies/actions: $s^{l}(t)=x_{c_{1}}^{l}(t)$, strategy to choose a WiFi channel $c_{1}$ for $\mathrm{WiFi}$ link $l$ from the set of available channels in $\mathcal{C}^{w}$.

- Utility function: To ensure a realistic representation of the game, we use the worst SIR values perceived by the two radio interfaces, WiFi and ZigBee, as utility function.

Hereafter, we describe the SIR given in Equation (3) that we extend to consider interfering transmitters using different technologies. It is worth noting that Equation (5) can be easily extended to more than two radio technologies, considering further for example Bluetooth. However, to simplify the analysis we conduct the study with only two components, corresponding to WiFi and ZigBee, respectively. Whence, the SIR of the player $l \in \mathcal{L}^{w}$, considering the WiFi interface, is given by:

$$
\operatorname{SIR}^{w}\left(x_{c_{1}}^{l}\right)(t)=10 \log \left(\frac{g_{l l} p_{w}^{l}}{I_{c_{1}}^{w}\left(x_{c_{1}}^{l}\right)+I^{w}\left(x_{c_{1}}^{l}\right)+I^{w z}\left(x_{c_{1}}^{l}\right)}\right),
$$

where

$I_{c_{1}}^{w}\left(x_{c_{1}}^{l}\right)$ : Co-channel interference from WiFi links of other sub-BBNs $\left(b_{k l}=0\right)$ sharing WiFi channel $c_{1}$ with WiFi link $l$.

$$
I_{c_{1}}^{w}\left(x_{c_{1}}^{l}\right)=\sum_{\substack{k \in \mathcal{L}^{w} \\ b_{k l}=0}} x_{c_{1}}^{l} x_{c_{1}}^{k} g_{l k} p_{w}^{k}
$$

$I^{w}\left(x_{c_{1}}^{l}\right)$ : Mutual interference from WiFi links of other sub-BBNs $\left(b_{k l}=0\right)$ using WiFi 
channels that overlap with $c_{1}$.

$$
I^{w}\left(x_{c_{1}}^{l}\right)=\sum_{\substack{k \in L^{w} \\ b_{k l}=0}}\left(\sum_{\substack{c \in C^{w} \\ c \neq c_{1}}} w_{c_{1} c} x_{c_{1}}^{l} x_{c}^{k}\right) g_{l k} p_{w}^{k}
$$

$I^{w z}\left(x_{c_{1}}^{l}\right)$ : Cross-interference from ZigBee links, using ZigBee channels other than $c_{2}$, overlapping with $c_{1}$

$$
I^{w z}\left(x_{c_{1}}^{l}\right)=\sum_{\substack{k \in L^{z} \\ k \neq h}}\left(\sum_{c \in C^{z}} a_{c_{1} c} x_{c_{1}}^{l} y_{c}^{k}\right) g_{l k} p_{z}^{k}
$$

$g_{l l}$ is the channel gain of link $l, g_{l k}$ the link gain from the transmitter $k$ to the receiver $l, p_{w}^{k}$ and $p_{z}^{k}$ are the WiFi and ZigBee transmit power, respectively.

Note that in expression (8) we use the binary parameter $a_{c_{1} c_{2}}$ to model the crosstechnology interference instead of the fractional $w_{c_{1} c_{2}}$ used in Equation (7) for mutual WiFi interference. In fact, although in the literature the interference of the IEEE $802.11 \mathrm{~b}$ has been modeled as an additive white Gaussian noise (AWGN) to the ZigBee signal, the experimental results performed in [29] show a significant performance degradation for ZigBee links in the presence of WiFi transmissions. Specifically, the authors measured a packet loss of $99,75 \%$ up to $100 \%$ in WBANs used for blood analysis and ECG sensing when a video streaming is executed over an interfering WiFi channel. Therefore, due to the tight constraints on WBANs' transmissions reliability, we consider the worst effect caused by WiFi interference on ZigBee communications, using the binary parameter $a_{c_{1} c_{2}} \in\{0,1\}$.

\section{1) Convergence of BBN-stage game: Nash Equilibrium}

Having defined the BBN stage of the SIM game, we then demonstrate that such game indeed admits at least one pure-strategy Nash equilibrium. Thus, we first define the utility function of player $l$ as follows:

$$
U_{w}\left(x_{c_{1}}^{l}\right)=10 \log \left(g_{l l} p_{w}^{l}\right)-10 \log \left(I F_{l}^{w}\left(x_{c_{1}}^{l}\right)\right)
$$

where $I F_{l}^{w}\left(x_{c_{1}}^{l}\right)$, denoted as the WiFi Interference Function of player $l$, is the total interference suffered by link $l$ when playing strategy $x_{c_{1}}^{l}$, and is expressed as follows: 


$$
\begin{aligned}
I F_{l}^{w}\left(x_{c_{1}}^{l}\right) & =I_{c}^{w}\left(x_{c_{1}}^{l}\right)+I^{w}\left(x_{c_{1}}^{l}\right)+I^{w z}\left(x_{c_{1}}^{l}\right) \\
& =\sum_{k \in W_{l} \cap \mathcal{L}^{w}} \sum_{c \in \mathcal{C}^{w}} f\left(x_{c_{1}}^{l}, x_{c}^{k}\right)+\sum_{j \in W_{l} \cap \mathcal{L}^{z}} \sum_{\substack{c \in C^{z} \\
c \neq c_{2}}} g\left(x_{c_{1}}^{l}, y_{c}^{j}\right)
\end{aligned}
$$

or function of the strategies:

$$
I F_{l}^{w}\left(s^{l}\right)=\sum_{k \in W_{l} \cap \mathcal{L}^{w}} f\left(s^{l}, s^{k}\right)+\sum_{j \in W_{l} \cap \mathcal{L}^{z}} g\left(s^{l}, s^{j}\right)
$$

where:

$$
f\left(s^{l}, s^{k}\right)= \begin{cases}0, & s^{l} \neq s^{k} \text { and WiFi channel } c_{1} \text { of link } l \\ & \text { does not overlap with WiFi channel of link } k . \\ g_{l k} p_{w}^{k}, & s^{l}=s^{k} \\ w_{c_{1} c} g_{l k} p_{w}^{k}, & s^{l} \neq s^{k} \text { and WiFi channel } c_{1} \text { of link } l \\ & \text { overlaps with WiFi channel of link } k .\end{cases}
$$

and:

$$
g\left(s^{l}, s^{j}\right)= \begin{cases}0, & \text { WiFi channel } c_{1} \text { of link } l \text { does not overlap } \\ & \text { with ZigBee channel of link } j . \\ g_{l j} p_{z}^{j}, & \text { WiFi channel } c_{1} \text { of link } l \text { overlaps with } \\ & \text { ZigBee channel of link } j .\end{cases}
$$

We observe that the maximization of utility function $U_{w}$ corresponds to the minimization of the Interference Function $I F^{w}$. Due to the property of monotone transformation, if the modified game with utility $I F^{w}$ is a potential game, then the original BBN-stage SIM game with utility $U_{w}$ is also a potential game with the same potential function. Then, the BBN-stage SIM game $\left(\mathcal{G}_{1}\right)$ is expressed as follows:

$$
\begin{aligned}
& \quad\left(\mathcal{G}_{1}\right): \min _{x_{c_{1}}^{l} \in S^{l}(t)} I F_{l}^{w}\left(x_{c_{1}}^{l}, x_{c_{1}}^{-l}\right) \quad \forall l \in \mathcal{L}^{w} \\
& \text { s.t. } \sum_{c \in \mathcal{C}^{w}} x_{c}^{l}=1 \quad \forall l \in \mathcal{L}^{w}(t) \\
& \quad x_{c_{1}}^{l} \in\{0,1\} \quad \forall l \in \mathcal{L}^{w}(t), c_{1} \in \mathcal{C}^{w},
\end{aligned}
$$

For convenience, we designate by $-l$ all the players belonging to $W_{l}$. Constraint (11) forces the assignment of a single WiFi channel for a single WiFi link for each player, the connectivity within the sub-BBNs is ensured so that a unique WiFi channel is allocated 
to every pair of links belonging to the same sub-BBN through the exchange of polling messages between the delegate player and the other players of each sub-BBN. The convergence of the BBN-stage SIM game to a Nash equilibrium is given by the following theorem:

Theorem 1: The BBN-stage SIM game $\mathcal{G}_{1}$ is an exact potential game.

Proof: we construct the potential function as follows:

$$
\Phi^{w}\left(s^{i}, s^{-i}\right)=\frac{1}{2} \sum_{i \in L^{w}} \sum_{k \in W_{i} \cap L^{w}} f\left(s^{i}, s^{k}\right)+\sum_{i \in L^{w}} \sum_{j \in W_{i} \cap L^{z}} g\left(s^{i}, s^{j}\right)
$$

Therefore, when player $l \in \mathcal{L}^{w}$ changes its action at time epoch $t \in \mathcal{T}$, from $s^{l}$ to $\hat{s}^{l}$, the variation of the potential function subsequent to this player's strategy change is given by:

$$
\begin{aligned}
& \Phi^{w}\left(s^{l}, s^{-l}\right)-\Phi^{w}\left(\hat{s}^{l}, s^{-l}\right)= \\
& \frac{1}{2} \sum_{\substack{i \in L^{w} \\
i \neq l}} \sum_{\substack{k \in W_{i} \cap L^{w} \\
w}} f\left(s^{i}, s^{k}\right)+\sum_{\substack{i \in L^{w} \\
i \neq l}} \sum_{j \in W_{i} \cap L^{z}} g\left(s^{i}, s^{j}\right)
\end{aligned}
$$

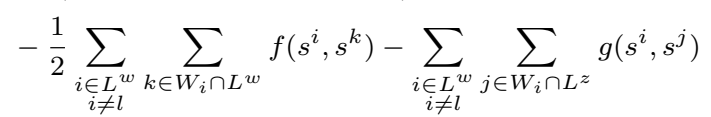

$$
\begin{aligned}
& +\frac{1}{2} \sum_{\substack{i \in L^{w} \\
i \neq l}} f\left(s^{i}, s^{l}\right)-\frac{1}{2} \sum_{\substack{i \in L^{w} \\
i \neq l}} f\left(s^{i}, \hat{s}^{l}\right) \quad(k=l) \\
& +\frac{1}{2} \sum_{k \in W_{l} \cap L^{w}} f\left(s^{l}, s^{k}\right)+\sum_{j \in W_{l} \cap L^{z}} g\left(s^{l}, s^{j}\right) \quad(i=l) \\
& -\frac{1}{2} \sum_{k \in W_{l} \cap L^{w}} f\left(\hat{s}^{l}, s^{k}\right)-\sum_{j \in W_{l} \cap L^{z}} g\left(\hat{s}^{l}, s^{j}\right) \quad(i=l)
\end{aligned}
$$

We can easily see that $(14)+(15)=0$. On the other hand, since each player has only interference with his neighboring set, then $\left\{i \in L^{w}: i \neq l\right\}=\left\{k \in W_{l} \cap L^{w}\right\}$, and we assume that function $f$ is symmetric so as we consider symmetric channel gains $\left(g_{l k}=g_{k l}\right.$ if $b_{k l}=0$, Fig.6), therefore:

$$
\begin{aligned}
\Phi^{w}\left(s^{l}, s^{-l}\right)-\Phi^{w}\left(\hat{s}^{l}, s^{-l}\right)= & \\
& \sum_{k \in W_{l} \cap L^{w}} f\left(s^{l}, s^{k}\right)+\sum_{j \in W_{l} \cap L^{z}} g\left(s^{l}, s^{j}\right) \\
& -\sum_{k \in W_{l} \cap L^{w}} f\left(\hat{s}^{l}, s^{k}\right)-\sum_{j \in W_{l} \cap L^{z}} g\left(\hat{s}^{l}, s^{j}\right) \\
& =I F_{l}^{w}\left(s^{l}, s^{-l}\right)-I F_{l}^{w}\left(\hat{s}^{l}, s^{-l}\right)
\end{aligned}
$$


Accordingly we prove that, when a delegate $l \in \mathcal{L}^{w}$ deviates from a strategy $s^{l}$ to an alternate strategy $\hat{s}^{l}$, the change in the exact potential function $\Phi^{w}$ exactly mirrors the change in l's utility. Therefore the BBN-stage SIM game is an exact potential game.

Thereby, we can rely on the following theorem [30] to confirm the existence of a Nash equilibrium to our game.

Theorem 2: Every potential game has at least one pure Nash equilibrium, namely the strategy $s^{l}$ that minimizes $\Phi^{w}\left(s^{l}\right)$.

The result of Theorem 2 motivates us to design the Best Response SIM algorithm in section 5 to resolve the BBN-stage SIM game.

\subsection{WBAN-stage SIM Game}

We now consider the WBAN-stage game, where each WBAN will be assigned a ZigBee channel to his ZigBee radio interface, that guarantees the minimal interference with his neighbors.

\section{1) ZigBee local interaction game}

Similarly to the BBN stage, denote $Z_{h}$ as the set of neighbors of ZigBee link $h$, including the set of edges between ZigBee link $h$ and interfering WiFi and ZigBee links, using the conflict graph:

$Z_{h}(t)=\left\{j \in L^{z}:(h, j) \subset E_{c}(t)\right\} \cup\left\{k \in L^{w}(t):(h, k) \subset E_{c}(t)\right\}$

Hence, we can define the local interaction game of the WBAN stage $\left(\mathcal{G}_{2}\right)$ as follows:

- Players: set $\mathcal{N}$ of WBANs. For the WBAN-stage, the player is assimilated to his ZigBee link $h$.

- Strategies/actions: $s^{h}(t)=y_{c_{2}}^{h}(t)$, strategy to choose a ZigBee channel $c_{2}$ for ZigBee link $h$ from the set of available channels in $\mathcal{C}^{z}$.

- Utility function: is, similarly to BBN stage, function of the SIR considering the ZigBee interface which is used for intra-WBAN communications, given by:

$$
\operatorname{SIR}^{z}\left(y_{c_{2}}^{h}\right)(t)=10 \log \left(\frac{g_{h h} p_{z}^{h}}{I^{w z}\left(y_{c_{2}}^{h}\right)+I^{z}\left(y_{c_{2}}^{h}\right)}\right),
$$


$I^{w z}\left(y_{c_{2}}^{h}\right)$ represents the cross-technology interference caused by mobile terminals using WiFi channels that interfere with the ZigBee channel $c_{2}$ on which WBAN link $h$ is tuned.

$$
I^{w z}\left(y_{c_{2}}^{h}\right)=\sum_{\substack{k \in \mathcal{L}^{w} \\ b_{k l}=0}} \sum_{c \in \mathcal{C}^{w}} a_{c c_{2}} x_{c}^{k} y_{c_{2}}^{h} g_{h k} p_{w}^{k}(t) .
$$

$I^{z}\left(y_{c_{2}}^{h}\right)$ accounts for the co-channel interference of nearby WBANs sharing the same ZigBee channel $c_{2}$ of player $h$.

$$
I^{z}\left(y_{c_{2}}^{h}\right)=\sum_{k \in \mathcal{L}^{z}} y_{c_{2}}^{k} y_{c_{2}}^{h} g_{h k} p_{z}^{k}(t) .
$$

Conversely to the BBN stage (Equation (5)), in Equation (22) only cross and cochannel interference components are considered at the denominator, since all ZigBee channels are completely orthogonal among each other, i.e. no mutual interference is there. In case of sharing the same ZigBee channel, i.e., expression (24), the corresponding experimental scenario in [29] measures $18 \%$ of packet losses, which led to the conclusion that the impact of ZigBee co-channel interference may be significant. Therefore, we model our game so that selecting different and non-overlapping ZigBee channels for intraWBAN communications emerges as the best strategy for all players. Unlike BBN-stage game where a unique WiFi channel is required by a sub-BBN, in WBAN stage, WBANs of the same sub-BBN use different ZigBee channels for intra-WBAN communications. Yet, to ensure a fair sharing of available ZigBee resources within BBNs, we consider local interaction behaviors among players interacting within the same neighboring set, which is translated in the utility function by a local cooperation quantity as a tradeoff to the player selfish attitude. Thus, we define the utility function of player $h$ for the WBAN-stage game as follows:

$$
\begin{aligned}
U_{z}\left(y_{c_{2}}^{h}\right) & =\operatorname{SIR}^{z}\left(y_{c_{2}}^{h}\right)+\sum_{k \in Z_{h}} \operatorname{SIR}^{z}\left(y_{c}^{k}\right) \\
& =10 \log \left(g_{h h} p_{z}^{h}\right)+\sum_{k \in Z_{h}} 10 \log \left(g_{k k} p_{z}^{k}\right)-I F_{h}^{z}\left(y_{c_{2}}^{h}\right)
\end{aligned}
$$

where: $\quad I F_{h}^{z}\left(y_{c_{2}}^{h}\right)=I_{h}\left(y_{c_{2}}^{h}\right)+\sum_{k \in Z_{h}} I_{k}\left(y_{c_{2}}^{h}\right)$

and: $I_{k}\left(y_{c_{2}}^{h}\right)=10 \log \left(I^{w z}\left(y_{c}^{k}\right)+I^{z}\left(y_{c}^{k}\right)\right) \quad, \forall c \in C^{z}: y_{c}^{k}=1$

$I_{k}\left(s^{h}\right)$, with $s^{h}=y_{c_{2}}^{h}$, is the total interference suffered by link $k$ of a neighboring WBAN 
when link $h$ plays strategy $y_{c_{2}}^{h}$.

As in [31], using the monotone transformation property, the WBAN-stage SIM game is expressed as follows:

$$
\begin{aligned}
& \left(\mathcal{G}_{2}\right): \min _{y_{c_{2}}^{h} \in S^{h}(t)} I F_{h}^{z}\left(y_{c_{2}}^{h}, y_{c_{2}}^{-h}\right) \quad \forall h \in \mathcal{L}^{z} \\
\text { s.t. } & \sum_{c \in \mathcal{C}^{z}} y_{c}^{h}=1 \quad \forall h \in \mathcal{L}^{z}(t) \\
& y_{c}^{h} \in\{0,1\} \quad \forall h \in \mathcal{L}^{z}, c \in \mathcal{C}^{z}
\end{aligned}
$$

Constraint (26) forces the assignment of a single ZigBee channel for a ZigBee link, for each player.

2) Convergence of WBAN-stage game: Nash Equilibrium

The property of the proposed local interaction game is characterized by the following theorem:

Theorem 4: $\mathcal{G}_{2}$ is an exact potential game which has at least one pure strategy NE, and the optimal solution of its potential function constitutes a pure strategy NE.

Proof: we construct the potential function as follows:

$$
\Phi^{z}\left(s^{h}, s^{-h}\right)=\sum_{k \in L^{z}} I_{k}\left(s^{h}, s^{-h}\right)
$$

if we compute the variation of the utility function when player $h \in \mathcal{L}^{z}$ changes its action at time epoch $t \in T$, from $s^{h}$ to $\hat{s}^{h}$, we obtain:

$$
\begin{aligned}
& I F_{h}^{z}\left(s^{h}, s^{-h}\right)-I F_{h}^{z}\left(\hat{s}^{h}, s^{-h}\right)= \\
& I_{h}\left(s^{h}, s^{-h}\right)-I_{h}\left(\hat{s}^{h}, s^{-h}\right)+\sum_{k \in Z_{h}}\left[I_{k}\left(s^{h}, s^{-h}\right)-I_{k}\left(\hat{s}^{h}, s^{-h}\right)\right]
\end{aligned}
$$

On the other hand, the variation of the potential function subsequent to this player's strategy change is given by: 


$$
\begin{aligned}
& \Phi^{z}\left(s^{h}, s^{-h}\right)-\Phi^{z}\left(\hat{s}^{h}, s^{-h}\right)= \\
& \sum_{k \in L^{z}} I_{k}\left(s^{h}, s^{-h}\right)-\sum_{k \in L^{z}} I_{k}\left(\hat{s}^{h}, s^{-h}\right)= \\
& I_{h}\left(s^{h}, s^{-h}\right)-I_{h}\left(\hat{s}^{h}, s^{-h}\right)+\sum_{k \in Z_{h}}\left[I_{k}\left(s^{h}, s^{-h}\right)-I_{k}\left(\hat{s}^{h}, s^{-h}\right)\right] \\
& +\sum_{\substack{k \in L^{z} \backslash Z_{h} \\
k \neq h}}\left[I_{k}\left(s^{h}, s^{-h}\right)-I_{k}\left(\hat{s}^{h}, s^{-h}\right)\right]
\end{aligned}
$$

Yet, with the local cooperative nature of WBAN-stage game, $h$ player's action only affects players in its interference range, thus we have:

$$
I_{k}\left(s^{h}, s^{-h}\right)-I_{k}\left(\hat{s}^{h}, s^{-h}\right)=0 \quad \forall k \in L^{z} \backslash Z_{h}, k \neq h
$$

This leads to the following equation:

$$
I F_{h}^{z}\left(s^{h}, s^{-h}\right)-I F_{h}^{z}\left(\hat{s}^{h}, s^{-h}\right)=\Phi^{z}\left(s^{h}, s^{-h}\right)-\Phi^{z}\left(\hat{s}^{h}, s^{-h}\right)
$$

Accordingly we prove that, when a player $h \in \mathcal{L}^{z}$ deviates from a strategy $s^{h}$ to an alternate strategy $\hat{s}^{h}$, the change in the exact potential function $\Phi^{z}$ exactly mirrors the change in $h$ 's utility.

Therefore the WBAN-stage SIM game is an exact potential game.

\subsection{A discussion on social interactions of WBANs in the SIM games}

The social information in the BBN and WBAN level games can be collected by using a signaling protocol, like one of those presented in $[27,32]$, to allow mobile terminals to exchange control messages (on proximity information) among each other in order to build (and maintain) the network topology and the conflict graph, and then compute in a completely distributed fashion the channel assignment that minimizes the (mutual and cross-technology) interference (or maximizes the SIR at $\mathrm{WiFi}$ and ZigBee radio interfaces), based on local information.

More in detail, we recall that our WiFi and ZigBee utility functions rely on the neighboring sets of a WBAN MT's WiFi and ZigBee pair of links $(l, h)$, 
defined as:

$$
\begin{aligned}
& W_{l}(t)=\left\{k \in L^{w}(t):(l, k) \subset E_{c}(t)\right\} \cup\left\{j \in L^{z}:(l, j) \subset E_{c}(t)\right\} \\
& Z_{h}(t)=\left\{j \in L^{z}:(h, j) \subset E_{c}(t)\right\} \cup\left\{k \in L^{w}(t):(h, k) \subset E_{c}(t)\right\}
\end{aligned}
$$

Link-state messages are used to spread topology information to the entire network. A link-state message contains two lists of WiFi and ZigBee neighbors, each identified by its WBAN and BBN identifiers. Such messages are used by the BBN players to build the network topology and the conflict graph. Then, WBANs' MTs send beacon messages to their neighbors, recognized in their neighboring sets $\left(W_{l}(t), Z_{h}(t)\right)$.

For example, a WiFi beacon message is only sent to the delegates of neighboring BBNs, since a single WiFi channel should be selected by each BBN. Such message contains the identifier of the WBAN, a list of neighbors (from which control traffic has been recently received), and his local information, needed for the utility functions of his neighbors, i.e., $x_{c_{1}}^{k}$ and $y_{c_{2}}^{j}$, where $c_{1}$ and $c_{2}$ are the WiFi and ZigBee channels selected by his WiFi and ZigBee links $(k, j)$. In contrast, the ZigBee beacon message is sent to his neighboring WBANs, within the same BBN, evenly, and contains in addition his SIRz value needed by the local interaction game, as explained hereafter.

Upon receiving a beacon message, the interference mitigation algorithm (BR-SIM) extracts the information necessary to update the utility function. In particular, for each WBAN receiving a ZigBee beacon message from a neighboring WBAN, BR-SIM extracts the SIRz advertised in the beacon message, and updates his utility function, by adding this SIRz value to the local cooperation quantity, as a tradeoff to the player selfish attitude (Equation (25)). For a detailed description of the information exchange protocol, please refer to our previous work [27]. 


\section{Best-Response algorithm for SIM game (BR-SIM)}

Potential games have two appealing properties: they admit at least one pure-strategy NE which can be obtained through a best-response dynamics carried out by each player, and they have the Finite Improvement Property (FIP) [33], which ensures the convergence to a NE within a finite number of iterations. In the following, we propose an iterative algorithm (Algorithm 1) that implements a best response dynamics for our proposed game.

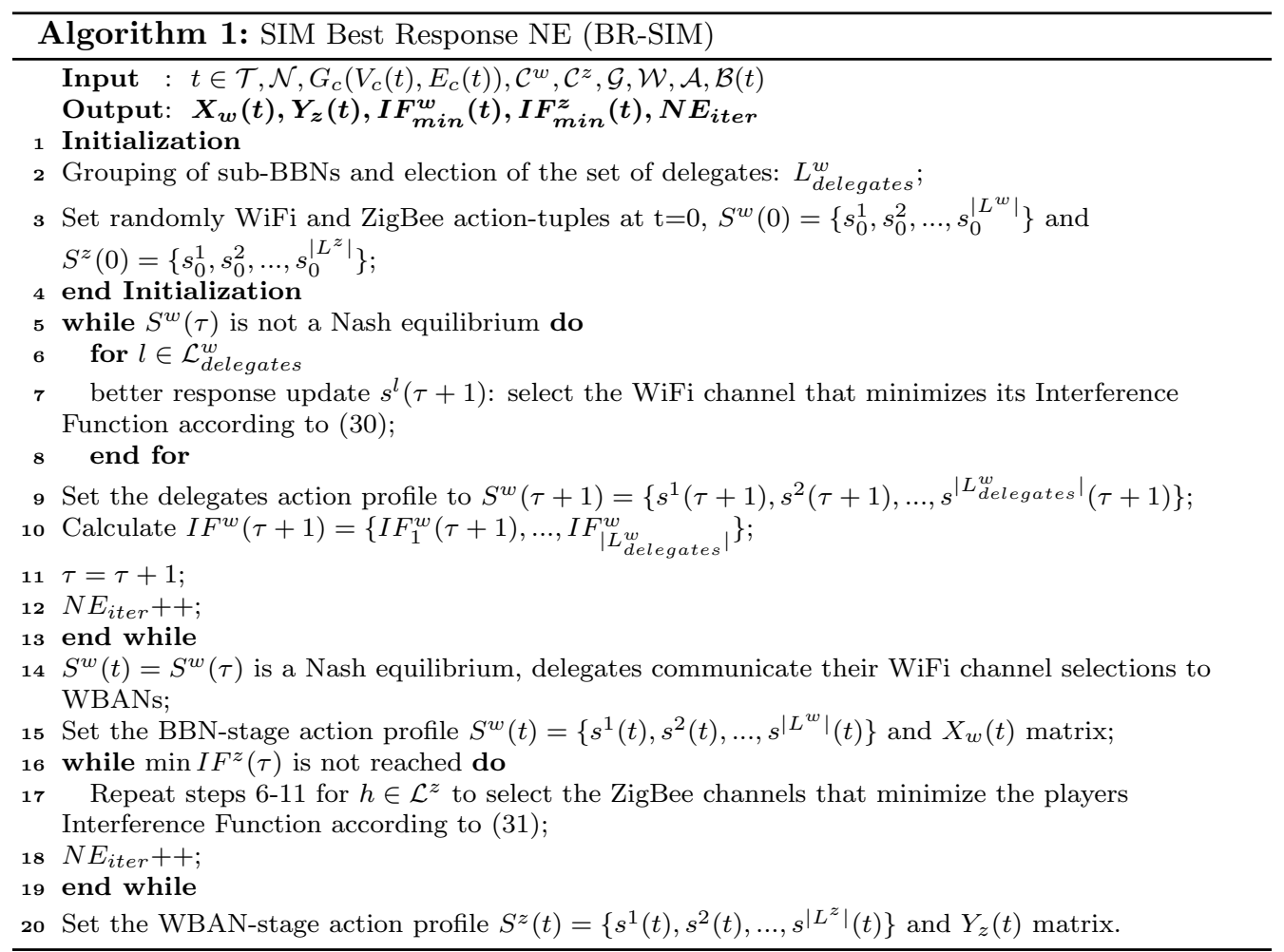

Algorithm 1 takes as input the current time epoch $t \in \mathcal{T}$, the set $\mathcal{N}$ of WBANs, the conflict graph $G_{c}\left(V_{c}(t), E_{c}(t)\right)$, the available WiFi and ZigBee channels $\left(\mathcal{C}^{w}, \mathcal{C}^{z}\right)$, the channel gain, the mutual and cross-technology channel overlapping, and the network connectivity matrices $(\mathcal{G}, \mathcal{W}, \mathcal{A}, \mathcal{B}(t))$. It gives as output the channel allocation matrices $X_{w}(t)$ and $Y_{z}(t)$, the minima of the WiFi and ZigBee Interference Functions obtained at the Nash Equilibrium, and the number of iterations $N E_{\text {iter }}$ needed to converge to a NE point. 
Algorithm 1 starts by forming the coalitions of sub-BBNs whose delegates are representative WiFi links situated in the center with symmetric gains. The delegates and the underlying WBANs are initialized to random WiFi and ZigBee channels with respect to the connectivity criterion within BBNs. Then, the algorithm iteratively examines whether there exists any player that is unsatisfied, and in such case a greedy selfish step is taken so that such player $l$ changes his current strategy $s^{l}(\tau), \tau<t$, to a better strategy $s^{l}(\tau+1)$ with respect to the current action profile of all other players, as follows:

$$
\begin{aligned}
& s^{l}(\tau+1)=\underset{s^{l} \in C^{w}}{\arg \min } I F_{l}^{w}\left(s^{l}, s^{-l}\right) \quad \text { s.t. } \\
& s^{-l}=\left\{s^{1}(\tau+1), s^{2}(\tau+1), \ldots, s^{l-1}(\tau+1), s^{l+1}(\tau), \ldots, s^{\left|L^{w}(t)\right|}(\tau)\right\}
\end{aligned}
$$

where $s^{1}, s^{2} \ldots, s^{l-1}$ have been updated to their best-responses at iteration $\tau+1$ and do not change from their selected strategies during the current iteration.

Alike the WiFi Best-response procedure, players iteratively update the ZigBee channels that minimize their Interference Functions, with respect to their WiFi channels selected at the BBN- (or WiFi-) stage step. Thus, for a ZigBee player $h$, the strategy domain of the ZigBee channel selection process is delimited to the set of available ZigBee channels $C_{h}^{z}(t)$, i.e., not overlapping with his assigned WiFi channel at time epoch $t$. Therefore, the best-response strategy of ZigBee player $h$ is expressed by:

$$
\begin{aligned}
& s^{h}(\tau+1)=\underset{s^{h} \in C_{h}^{z}(t)}{\arg \min } I F_{h}^{z}\left(s^{h}, s^{-h}\right) \quad \text { s.t. } \\
& s^{-h}=\left\{s^{1}(\tau+1), s^{2}(\tau+1), \ldots, s^{h-1}(\tau+1), s^{h+1}(\tau), \ldots, s^{\left|L^{z}(t)\right|}(\tau)\right\}
\end{aligned}
$$

Due to the FIP property, such algorithm is guaranteed to converge in a finite number of iterations to a BBN-stage NE, and then to a local interaction ZigBee NE where no player has an incentive to deviate from his best-response choice.

\section{Sub-Optimal Randomized Trials for SIM game (SORT-SIM)}

In large-scale networks with several BBNs, especially in real-time-constrained applications, the exhaustive search of NE can be extremely time consuming. Therefore, we propose, as an alternative solution, the SORT-SIM algorithm to deal with this specific 
issue. SORT-SIM is based on the principle of ensuring feasible SIR values for all players while allowing them to play simultaneously, and reducing the probability of channel selection conflicts.

Algorithm 2 takes the same inputs as Algorithm 1, and gives the same outputs, i.e., the channel allocation matrices $X_{w}(t)$ and $Y_{z}(t)$, the minima of the Interference Functions, and the number of iterations $S O R T_{i t e r}$ needed to reach the sub-optimal solution.

At the beginning, Algorithm 2 describes the main steps relative to the grouping of subBBNs, the election of their representative links and the calculation of their corresponding set of neighbors. Then, the WiFi channel allocation is performed, for each delegate $l$, as follows:

i. First, select randomly a WiFi channel from the list of free WiFi channels, if available, i.e., not allocated in neighboring set of link $l$ (step 8).

$$
C_{\text {free }}^{w}(l)=\left\{c \in \mathcal{C}^{w}: \forall k \in \mathcal{W}_{l}(t) \cap \mathcal{L}^{w}(t), x_{c}^{k}=0\right\}
$$

ii. If no free channel is available, calculate at step 9 the utility $\left(S I R^{w}\right)$ for each delegate and select randomly from the list, WiFi channels that provide an $S I R^{w}$ above the threshold value $\left(S I R_{t h}^{w}\right)$.

$$
c_{1}=\left\{\begin{array}{l}
\operatorname{Rand}\left(C_{\text {free }}^{w}(l)\right), \text { if } \mathcal{C}_{\text {free }}^{w}(l) \neq \emptyset \\
\operatorname{Rand}\left\{c \in \mathcal{C}^{w}: \operatorname{SIR}^{w}\left(x_{c}^{l}\right)>S I R_{t h}^{w}\right\}, \text { otherwise }
\end{array}\right.
$$

iii. To ensure a fair sharing of resources, a WBAN should release his WiFi channel after at most $\theta$ s. $\theta$ is defined as the maximum time of reservation of the wireless channel, and is assumed as a configurable parameter.

iv. Finally, the WBANs belonging to the same sub-BBN are tuned on the $\mathrm{WiFi}$ channel selected by their leader.

The previous operations are iteratively repeated until reaching a number of trials where no WBAN has an incentive to deviate from his channel choice, presenting, thus, a sub-optimal solution for the SIM problem.

Since multiple ZigBee channels could be used within the same sub-BBN, the channel allocation problem is relaxed in the WBAN stage and the aforementioned operations are processed indifferently for each ZigBee link $h \in \mathcal{L}^{z}(t)$, omitting the last operation (iv.), except some restrictions on the available ZigBee channels. Indeed, for each sub-BBN 


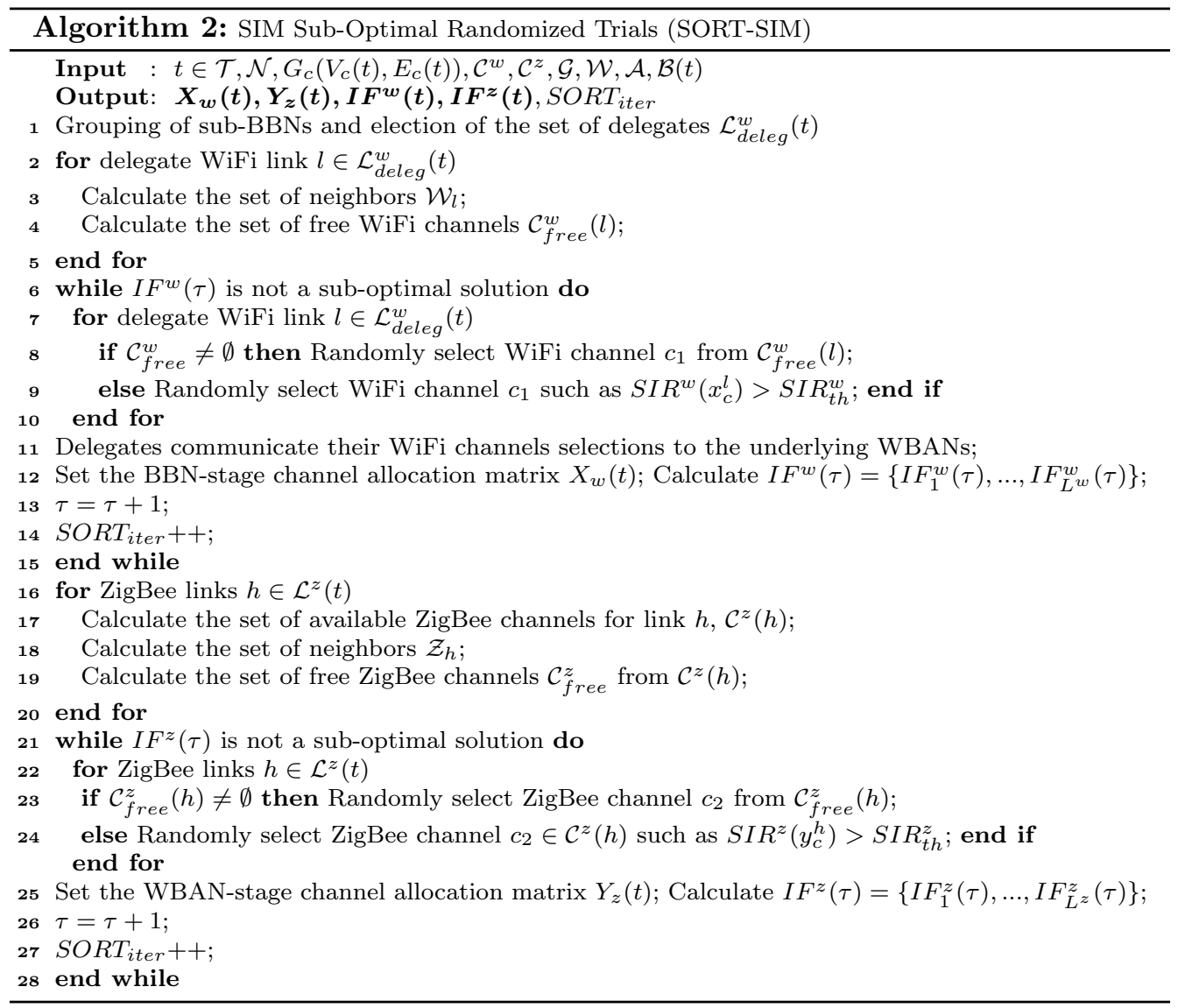


provided with WiFi channel $c_{1}$, we should delimit the set of available ZigBee channels $\mathcal{C}^{z}(h)$ eliminating those that overlap with $c_{1}$ :

$$
C^{z}(h)=\left\{c \in \mathcal{C}^{z}: a_{c c_{1}}=0\right\} \quad \forall(l, h) \subset \mathcal{L}(t), c_{1} \in \mathcal{C}^{w}: x_{c_{1}}^{l}=1
$$

Hence, the algorithm calculates the set of available ZigBee channels for each sub-BBN (step 17), as well as the list of free ZigBee channels (step 19), which is computed with respect to the set $\mathcal{C}^{z}(h)$.

$C_{\text {free }}^{z}(h)=\left\{c \in \mathcal{C}^{z}(h): \forall k \in \mathcal{Z}_{h} \cap \mathcal{C}^{z}, y_{c}^{k}=0\right\}$

Finally, the ZigBee channel $c_{2}$ is computed similarly to the WiFi part (step 23, 24), as follows:

$c_{2}=\left\{\begin{array}{l}\operatorname{Rand}\left(C_{f r e e}^{z}(h)\right), \text { if } \mathcal{C}_{f r e e}^{z}(h) \neq \emptyset \\ \operatorname{Rand}\left\{c \in \mathcal{C}^{z}(h): S I R^{z}\left(y_{c}^{h}\right)>S I R_{t h}^{z}\right\}, \text { otherwise. }\end{array}\right.$

We also keep the condition on the fair sharing of resources, so that a WBAN should release his ZigBee channel after at most $\theta$ s.

Although the proposed SORT-SIM algorithm does not provide the optimal solution for SIM game, it guarantees, at the worst cases, an appropriate strategy with feasible SIR value, i.e. $S I R>S I R_{t h}$, while reducing the probability to select the same channel by neighboring WBANs. Furthermore, the simplicity of implementation of SORT-SIM algorithm is a major feature for such highly constrained BBN environment.

\section{Performance Evaluation}

This section illustrates and discusses the numerical results obtained in different network scenarios of both algorithms BR-SIM and SORT-SIM, which have been implemented using the Scilab software package [34]. Then, we compare our algorithms with two existing power control approaches $[8,35]$, which handle almost the same problem we tackle in this work, i.e., the interference mitigation for nearby WBANs.

The mobile WBANs, which number varies in the range [20,50], are randomly deployed in a $1000 \times 1000 \mathrm{~m}^{2}$ area, and grouped into four overlapping BBNs. The mobility is simulated using the common random way-point model [36] (Fig.7). We consider the first five overlapping WiFi channels of the ISM band $\left(\mathcal{C}^{w}=\{1,5\}\right)$ and the whole band of 


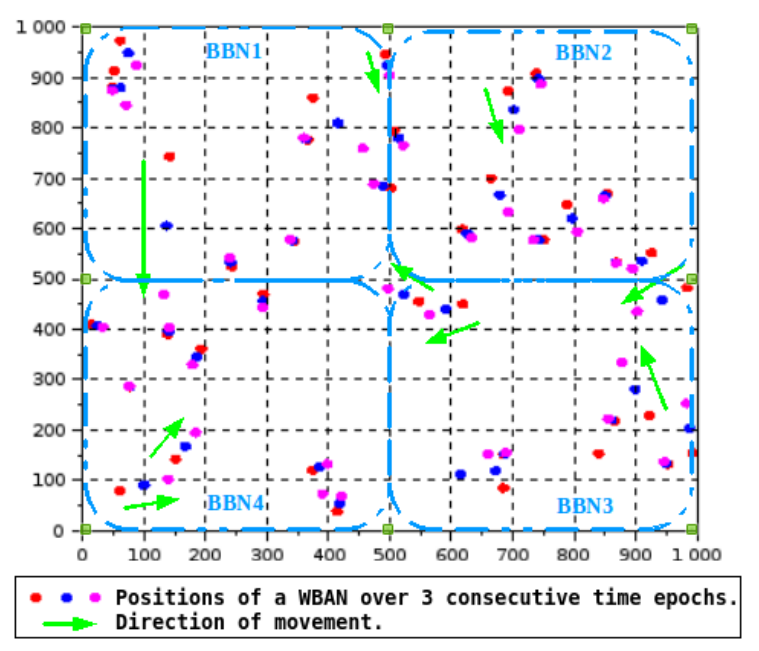

Figure 7: Simulation scenario for $\mathrm{N}=40 \mathrm{WBANs}$

ZigBee channels $\left(\mathcal{C}^{z}=\{11,26\}\right)$ in order to simulate the WiFi mutual interference and the cross-technology scenarios. To compute channel gains, we refer to the BBN-specific channel gain model in [23]. The WiFi and ZigBee transmission powers are set to $100 \mathrm{~mW}$ and $1 \mathrm{~mW}$, respectively. To prove and compare the effectiveness of our two distributed solutions, we successively evaluate the effect of the WBANs density on the dynamics of the BR-SIM channel selection algorithm and then on the performance of the SORT-SIM algorithm. More specifically, we evaluate the WiFi and ZigBee signal-to-interference ratios for each BBN, proving that the BR-SIM algorithm guarantees a fair sharing of wireless resources, while SORT-SIM presents quickness benefits in some BBN scenarios. SIRw and SIRz, in Equations (5) and (22), respectively, are indeed our original utility functions that are obtained after the computation of the WiFi and ZigBee Interference Functions.

\subsection{BR-SIM versus SORT-SIM}

The curves on Fig.8 and Fig.9 illustrate, respectively, the dynamics of the BR-SIM algorithm for different BBN densities, namely for the number of WBANs $\mathrm{N}=20$ and $\mathrm{N}=40$. More specifically, Fig.8a and Fig.8b show the average WiFi SIR and ZigBee SIR, respectively, for $N=20$. Fig.8c further shows the convergence of the SIR at the ZigBee interface of a subset of players under the BR-SIM algorithm. Similarly, Fig.9a, Fig.9b 


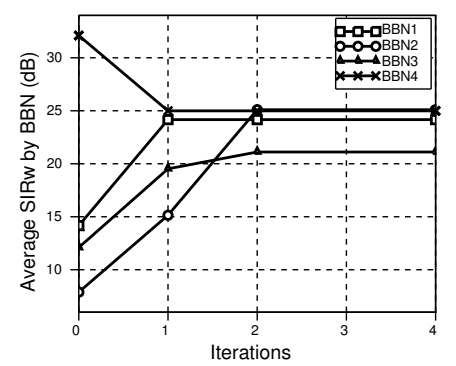

(a) Average WiFi SIR.

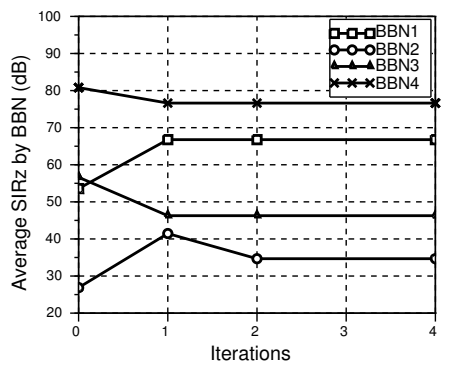

(b) Average ZigBee SIR.

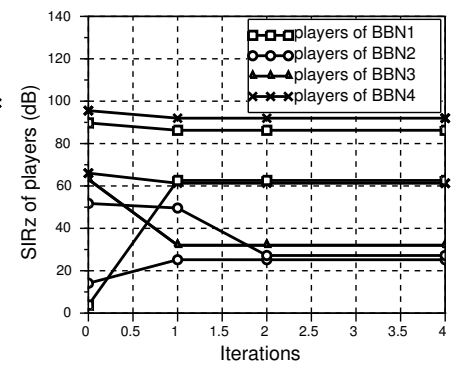

(c) SIRz of a subset of players.

Figure 8: Dynamics of the BR-SIM algorithm for each BBN, with $N=20$ WBANs

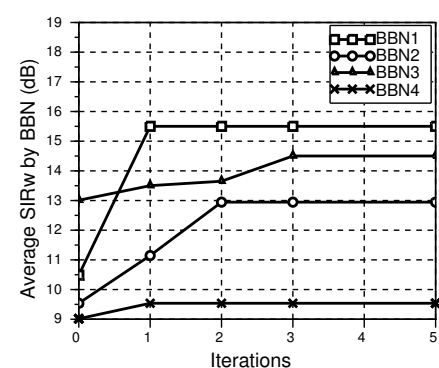

(a) Average WiFi SIR.

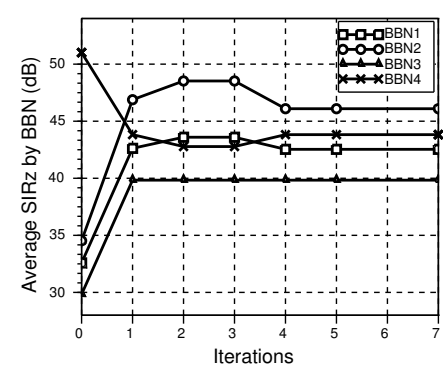

(b) Average ZigBee SIR.

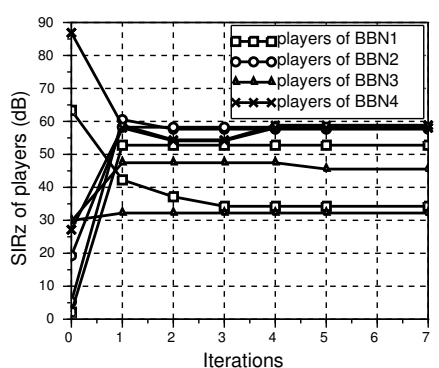

(c) SIRz of a subset of players.

Figure 9: Dynamics of the BR-SIM algorithm for each BBN, with $\mathrm{N}=40$ WBANs

and Fig.9c display, respectively, the evolution of the average SIR and the actual SIR values for a subset of players by each BBN, so as to show the effect of the network density on the convergence of the BR-SIM algorithm. As expected, increasing the BBN density results in increasing the network overall interference and the number of iterations to reach an equilibrium.

Besides, we notice at the Nash Equilibrium that the worst WiFi SIR $(21 \mathrm{~dB}$ for $\mathrm{N}=20$ and $9 \mathrm{~dB}$ for $\mathrm{N}=40$ ), measured with the standard transmission power of $20 \mathrm{dBm}$ $(100 \mathrm{~mW})$ is always above the receiver sensitivity of most commercial cards (the lowest receiver sensitivity for the Atheros chipset is $-95 \mathrm{~dB}$ ), even considering other effects like fading and thermal noise. The same conclusions are observed for the worst ZigBee SIR measured by all four BBNs (i.e., the WBAN that experiences the worst SIR in a BBN), which varies between 25 and $30 \mathrm{~dB}$ for $\mathrm{N}=20$ and $\mathrm{N}=40$ respectively. Note that the 


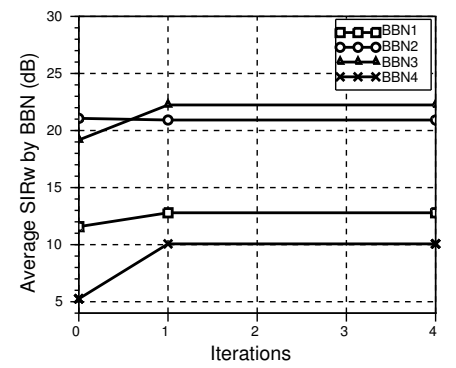

(a) Average WiFi SIR.

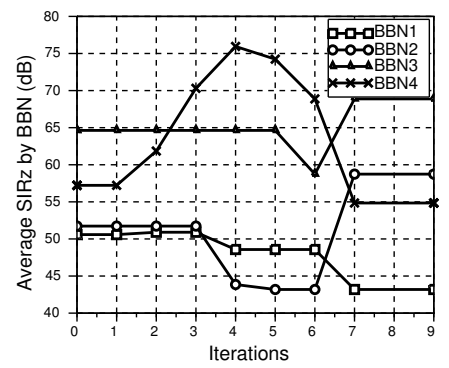

(b) Average ZigBee SIR.

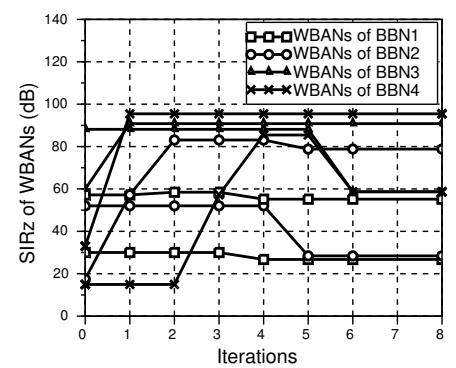

(c) SIRz of a subset of WBANs.

Figure 10: Iterations of the SORT-SIM algorithm for each BBN, with N=20 WBANs

worst SIR measured at the ZigBee interface is higher than the value measured at the WiFi interface due to the restricted number of overlapping WiFi channels used in the simulation in order to enable mutual and cross-technology interference, thus resulting in conflicting transmissions using the WiFi technology. Naturally, within a BBN only WiFi transmissions coming from surrounding BBNs are considered in the computation of the WiFi interference, since we assume the utilization of a coordination scheme for intra-BBN communications, whereas the ZigBee interface of any WBAN experiences both intra-BBN and inter-BBN interference. Thereby, further experiments with non-overlapping WiFi channels would reverse the previous conclusions and assess higher values of WiFi SIR versus ZigBee SIR.

Yet, the performance of BR-SIM is ensured since it provides a rather fair, sociallyaware channel allocation, so that both WiFi and ZigBee signal-to-interference ratios tend to be quite close to a mean value at the Nash Equilibrium. Nevertheless, a noticeable decrease in the range of SIR values (mainly SIRz), at the NE point, is observed when the density of the WBANs is high $(\mathrm{N}=40)$, and the SIR curves are tightly close. Indeed, higher densities occasion a more fair spreading of players over the neighboring BBNs, that will suffer from relatively fair interference environment. This explains why, for lower densities, the average SIR values for each BBN are spread out over a larger range of values.

On the other hand, Fig.10 and Fig.11 illustrate the signal-to-interference ratios at WiFi and ZigBee interfaces obtained by the SORT-SIM algorithm for the same topology 


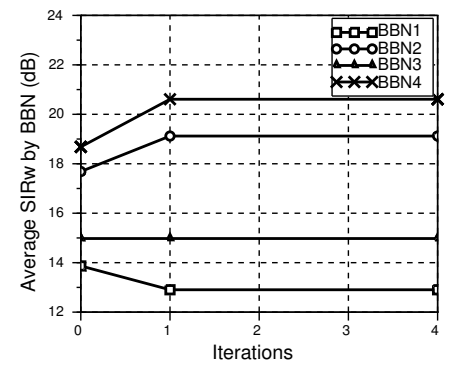

(a) Average WiFi SIR.

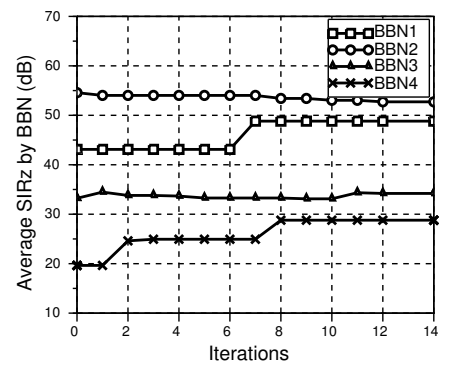

(b) Average ZigBee SIR.

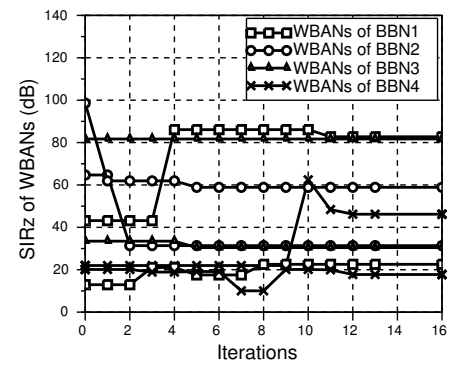

(c) SIRz of a subset of WBANs.

Figure 11: Iterations of the SORT-SIM algorithm for each BBN, with N=40 WBANs

configurations (i.e., $\mathrm{N}=20$ and $\mathrm{N}=40$ ). Almost the same conclusions can be made for SORT-SIM, as far as BR-SIM results, in terms of the evolution of SIR metrics as a function of WBANs density, wherein we can observe the degradation of both WiFi and ZigBee SIR values while increasing the BBN density. However, if we observe the average SIR of the whole network we can notice the main differences between the behaviour of the two algorithms. Indeed, Fig.16a and Fig.16b show a more accentuated steepness of SORT-SIM curves compared to that of BR-SIM, which means that the effectiveness of SORT-SIM is more density-sensitive, while BR-SIM seems to be more robust to density changes. In fact with higher densities, i.e., beyond $\mathrm{N}=30$ players, SORT-SIM presents more severe degradation in SIR values for both WiFi and ZigBee transmission links, whereas BR-SIM shows a smooth decrease while preserving good SIR ratios.

Now, if we observe the performance of each algorithm separately, we notice rather similar behaviours at low densities (Fig.8 and Fig.10), where few players are spread out over the simulation area. Both algorithms compete in allocating feasible, near optimal, WiFi and ZigBee channels to all players. However, for high densities we notice that BR-SIM curves merge around the average SIR, while SORT-SIM still presents great divergences among players' SIR values. This can be explained by the usefulness of the cooperative component of BR-SIM, where the local interactions among neighbors allow it to fairly share the wireless resources. Whereas, SORT-SIM proceeds in a completely non-cooperative manner, thus some players get maximal SIR values, while others settle for channel allocations with minimal SIR values, just above the threshold. 
Yet, the SIR values at both $\mathrm{WiFi}$ and ZigBee interfaces under the BR-SIM and SORT-SIM algorithms are illustrated in detail in Fig.12 and Fig.13, respectively. More specifically, these figures show the empirical Cumulative Distribution Function (CDF) of the SIR when the total number of WBANs $N=40$ and for a time duration of $300 \mathrm{~s}$, which is divided in 30 time epochs of $10 \mathrm{~s}$ each. Let us first focus on the SIR metric for WiFi obtained with BR-SIM (Fig.12a) and SORT-SIM (Fig.13a). It can be observed that the SIR values under both algorithms are quite similar and range from 0 to $\approx 40 \mathrm{~dB}$. However, it is not hard to see that BR-SIM guarantees for the majority of the players fair values of SIR (in the range $[10,25]$ ), while SORT-SIM performs WiFi channel assignment to transmission links in a much more aggressive way, where some players enjoy high values of SIR while others suffer from very low values. Similarly, for the SIR value measured at the ZigBee interface, Fig.12b and 13b show that in more than $50 \%$ of the scenarios, the SIR is higher than approximately $50 \mathrm{~dB}$. However, note that in the case of SORTSIM and for the 6 considered WBANs the percentage of players getting a value of SIR below $20 \mathrm{~dB}$ is larger than the one obtained with BR-SIM. Hence, this trend confirms the fact that BR-SIM guarantees at the same time some fairness along players and good performance.

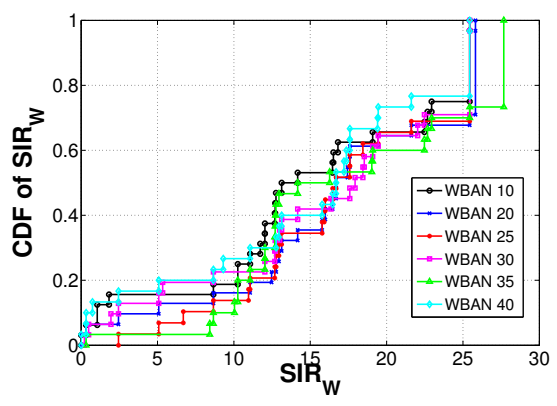

(a) CDF of WiFi SIR

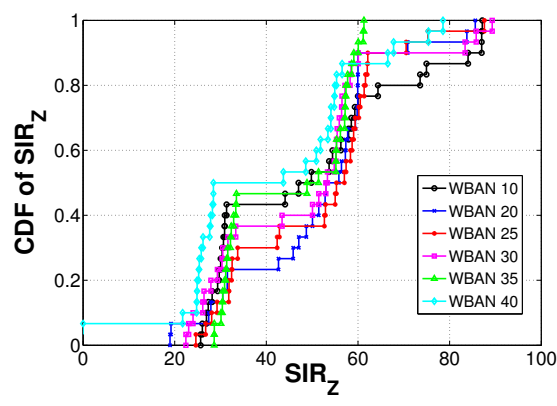

(b) CDF of ZigBee SIR

Figure 12: BR-SIM: Empirical Cumulative Distribution Function (CDF) of the SIR measured at WiFi and ZigBee interface of all WBANs in the BBN scenario of 40 WBANs with 30 time epochs of $10 \mathrm{~s}$ each.

Besides, we calculate with Scilab the computation time (CPU time) for both algorithms and we find noticeable difference between them. Indeed, the BR-SIM computation time is about four times larger than that of the SORT-SIM execution instance. For example, the maximum computation time we measured to solve the BR-SIM algorithm over 


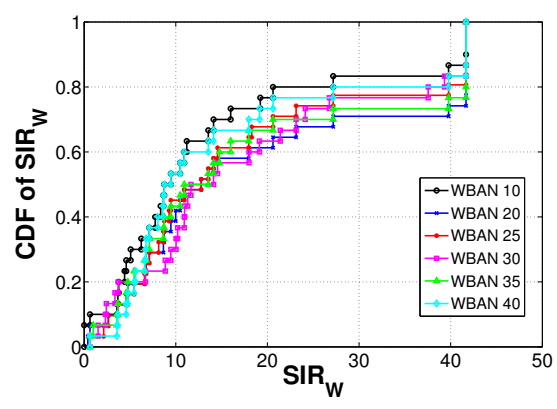

(a) CDF of WiFi SIR

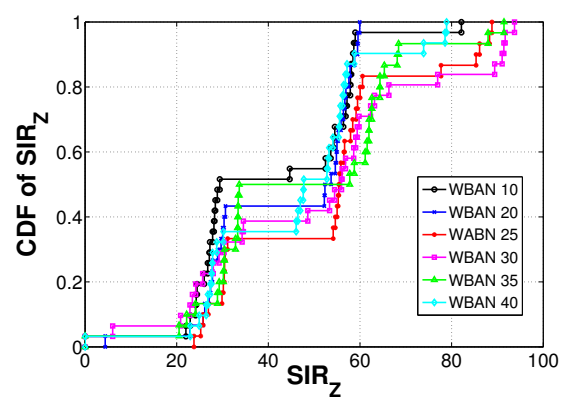

(b) CDF of ZigBee SIR

Figure 13: SORT-SIM: Empirical Cumulative Distribution Function of the SIR measured at WiFi and ZigBee interface of all WBANs in the BBN scenario of 40 WBANs with 30 time epochs of $10 \mathrm{~s}$ each.

30 consecutive time epochs was approximately equal to 1060 seconds, for $\mathrm{N}=50$ WBANs. Conversely, SORT-SIM takes less than 228 seconds to find the sub-optimal solutions for the SIM problem, under the same network instances and parameters' settings. Furthermore, it can be observed that the BR-SIM algorithm converges to a stable operational point in few iterations, in particular, all BBNs converge to their best WiFi and ZigBee channel allocations in at most 3 and 5 iterations, respectively, while SORT-SIM performs with greater number of iterations (up to 15), but within less computation time.

Finally, BR-SIM outperforms in terms of fairness and robustness the SORT-SIM algorithm, especially at higher densities, thus representing a practical solution for interference mitigation in realistic BBN scenarios. However, SORT-SIM presents simplicity and rapidity benefits which makes it useful, under specific BBN scenarios, mainly at low densities and low QoS requirements.

\subsection{Comparison with power control approaches}

In this section, we compare our BR-SIM and SORT-SIM algorithms to the distributed power control algorithm proposed in [8] and to the joint relay selection and transmit power control algorithm proposed in [35].

Authors of [8] formulated a power control game considering interference between neighboring WBANs and energy-efficiency. They derived a distributed power control algorithm, called the ProActive Power Update (PAPU) algorithm, to reach a unique Nash Equilibrium (NE) representing the best tradeoff between energy-efficiency and 
network utility. As in our model, PAPU assumes a TDMA-based MAC protocol to deal with intra-WBAN interference avoidance, and uses the SINR metric to define the utility function of the power control game. However, neither WBAN mobility is considered, nor wireless technologies are specified.

Alike our SIR metrics defined in our paper by expressions (5) and (22), respectively, for WiFi and ZigBee received signals, the SINR was defined in [8] without consideration of heterogeneous wireless technologies. This will be reflected in the final SINR values, as we will show hereafter.

Indeed, we have implemented the PAPU algorithm with the same network configuration of our BR-SIM and SORT-SIM algorithms, and with the following definition of the power best-response performed by each WBAN/player:

$$
b_{i}\left(p_{-i}\right)=\frac{1}{c_{i}}-\frac{\sum_{j \neq i} h_{j i} p_{j}+n_{0}}{h_{i i}}
$$

where $p_{j}$ is the transmission power of player $j, h_{j i}$ represents the channel gain between transmitter $j$ and receiver $i, h_{i i}$ the intra-network gain, $n_{0}$ is the background white noise power (which is ignored in our simulations since we calculate the SIR), and $c_{i}$ the power price. The obtained (average) SIR values are reported in Fig.14 and Fig.16.

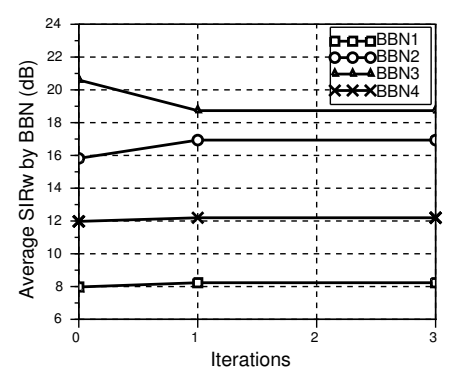

(a) Average WiFi SIR.

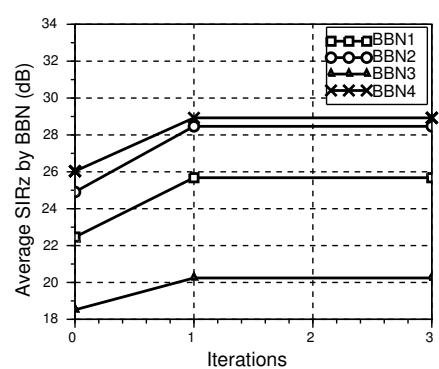

(b) Average ZigBee SIR.

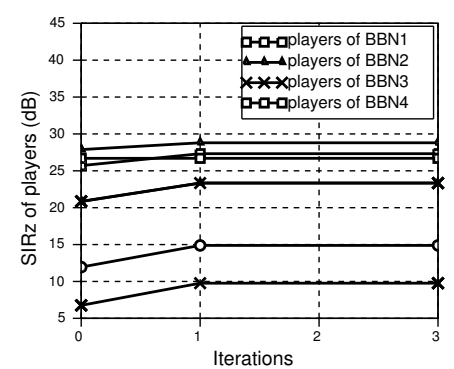

(c) SIRz of a subset of WBANs.

Figure 14: Dynamics of the PAPU algorithm for each BBN, with $N=40$ WBANs

First, it can be observed from Fig.14 that PAPU is rather efficient with respect to WiFi SIR maximization; results are almost in the same range as the BR-SIM and SORT-SIM algorithms. This can be explained by the fact that PAPU's WiFi SIR does not consider the cross-technology interference from ZigBee on WiFi links. Only intra- 
WBAN channel gains are involved, whereas in real BBN scenarios the cross-technology channel gains introduce further interference components to the SIR denominator.

However, the difference mainly appears in the second-stage game (Fig.16b), where PAPU provides less efficient SIR values for the ZigBee signal. Whilst BR-SIM and SORTSIM provide ZigBee SIR values over 20dB (up to 80dB), PAPU's maximum ZigBee SIR is around $20 \mathrm{~dB}$ (up to $40 \mathrm{~dB}$ for lower network densities). Yet, as its authors explained, PAPU requires limited information exchange between WBANs, and as a consequence the player strategy is purely selfish, without any consideration of neighboring WBANs' utilities. With local interactions of our SIM game, BR-SIM and SORT-SIM achieve better SIR values, and thus stronger wireless signal. This also explains the regularity of PAPU curves, whereas the negotiations among players are better observed on the BR-SIM and SORT-SIM curves.

It is worth noting that the reduced number of iterations of the PAPU algorithm within our network configuration, compared to that of the original paper, is also due to the local interaction behavior among players, which allows a rapid convergence to the NE.

We now compare BR-SIM and SORT-SIM to the joint Relay Selection and transmit Power Control algorithm, referred to hereafter as RSPC algorithm, proposed in [35].

In [35], each WBAN has the following configuration (see Fig.15): a hub at the chest, two relays at the right and left hips, and three sensors at other suitable locations. The hub, the sensor and the two relays are denoted as $H, S, R_{1}$ and $R_{2}$, respectively. Time division multiple access (TDMA) and asynchronous TDMA are respectively used as intra- and inter-WBAN access schemes, since it has been shown in [37] that they provide better interference mitigation than other access schemes in terms of power consumption and channel quality.

The major contribution of the RSPC algorithm is the use of opportunistic relaying with no cooperation between WBANs to provide inter-body channel gain measurements, in order to improve reliability (decrease the outage probability) and reduce the power consumption. RSPC uses the on-body and inter-body channel data sets in [38], obtained through exhaustive scenarios 


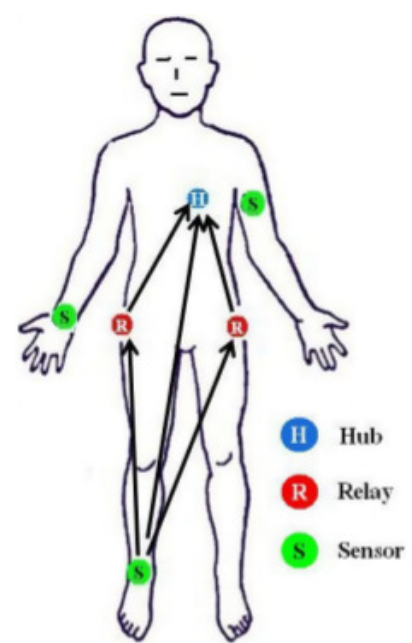

Figure 15: WBAN configuration for the RSPC algorithm [35].

performed in realistic environments, over several hours of normal everyday activities. In each experiment, sensors transmit in a round-robin fashion with 5 ms separation between each other.

Thereby, the RSPC algorithm can be summarized in the three following steps:

1. Power control at the sensor level: the sensor performs power control on a channel at time epoch $\tau$ using the value at time epoch $\tau-1$, and selects the one-hop relay: StoH (Sensor-to-Hub), StoR1 (Sensor-to-Relay1) or StoR2 (Sensor-to-Relay2).

2. Power control at the relay level: select the relay transmit power to the hub, in the transmit range.

3. Branch selection at the hub: the hub selects the path (StoH, StoR1R1toH or StoR2-R2toH) that gives the best SINR.

The authors of [35] assert that relay-assisted communications can reduce co-channel interference from neighboring WBANs, by increasing the SINR of the packets transmitted by the sensor node and received at the WBAN coordinator (the hub/the MT in our model), expressed by: 


$$
S I N R=\frac{T_{x} \times\left|h_{T x R x}\right|^{2}}{\sum T_{x_{\text {int }, i}}\left|h_{\text {int }, i}\right|^{2}}
$$

where $T_{x}$ is the sensor/relay transmit power obtained by the Power Control function (step 1 or 2 of the RSPC algorithm). $\left|h_{T x R x}\right|$ represents the average channel gain across the duration of the sensor/relay transmitted signal, while $\left|h_{i n t, i}\right|$ is the channel gain between the interferer int, which is the neighboring WBAN sensor, and the sensor or selected relay $i$. Finally, $T_{x_{\text {int }, i}}$ denotes the interfering power of neighboring WBAN sensor int to the sensor/relay $i$. The instantaneous noise at the receiving node has been omitted, since we compare SIR metrics.

For the one-hop relay selection, we consider the WBAN configuration given in Fig.15. Since TDMA is used as access scheme, sensors cannot transmit simultaneously within a WBAN. Yet, to adapt the RSPC algorithm to our network model, we focus on a WBAN's sensor-of-interest, and we assimilate the neighboring interferer sensor to its corresponding MT. The one-hop relay process will be considered while selecting the intra-WBAN transmit power, i.e. in the ZigBee stage. We further assume that WBANs use a WiFi channel for inter-WBAN exchanges. Power control will also be performed for WiFi transmissions in a way to maximize the MT WiFi SIR, using the ZigBee power vectors of neighboring WBANs, computed at the previous time epoch.

We run our simulations and we calculate the WBAN's SIR (SIRw and SIRz), considering the aggregate interference due to transmit powers of the neighboring WBANs.

It can be observed from Fig. 16a that, in general, the RSPC WiFi SIR curve lies between BR-SIM and SORT-SIM curves. Even though RSPC does not perform iterations to reach the best SIR, unlike the game models, it optimizes once the sensor/relay transmit power with its Power Control algorithm and achieves rather efficient SIR values. These results can be explained by analyzing, as we do hereafter in Fig. 17, the aggregate interference, calcu- 


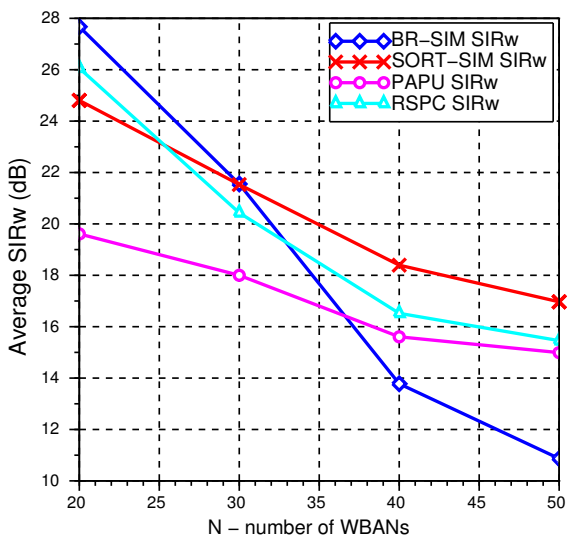

(a) Average WiFi SIR.

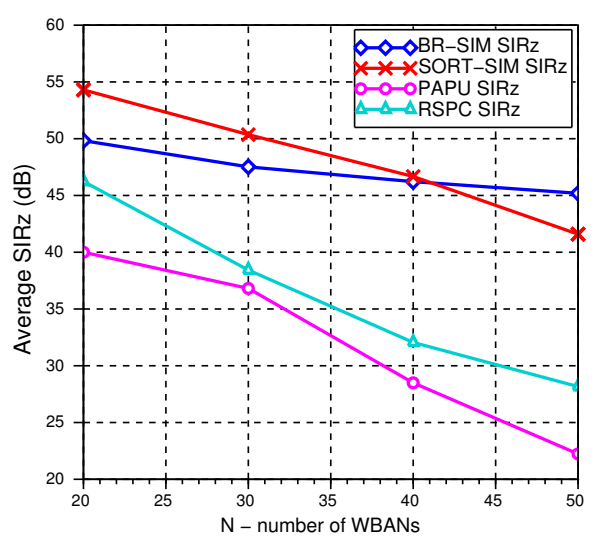

(b) Average ZigBee SIR.

Figure 16: BR-SIM and SORT-SIM vs PAPU and RSPC. Average WiFi and ZigBee SIR as a function of network density.

lated as the sum of interference suffered by the hub/MT, due to WiFi and ZigBee transmissions of neighboring WBANs.

In Fig. 17, we notice an important gap between the RSPC aggregate interference and the one obtained by our algorithms (BR-SIM and SORTSIM) and PAPU. Specifically, $I N_{B R-S I M}$ and $I N_{S O R T-S I M}$ are always lower than those of PAPU and RSPC, even though sometimes the WiFi SIR of RSPC is higher than the one achieved by BR-SIM or SORT-SIM (Fig. 16a). This can be explained as follows:

- The aggregate interference values of the BR-SIM and SORT-SIM algorithms are considerably lower than those of PAPU and RSPC, because in our interference mitigation model we assign $\mathrm{WiFi} / \mathrm{ZigBee}$ channels to wireless links in a way to reduce the co-channel and cross-interference components. Therefore, neighboring interfering WiFi/ZigBee links are omitted (by allocating them orthogonal channels) or reduced by the $w_{m n}$ scalar, to ensure minimum mutual interference.

- The gap is less important for the SIR values, because the MT/Hub channel gains and transmit powers are far larger than the interference 


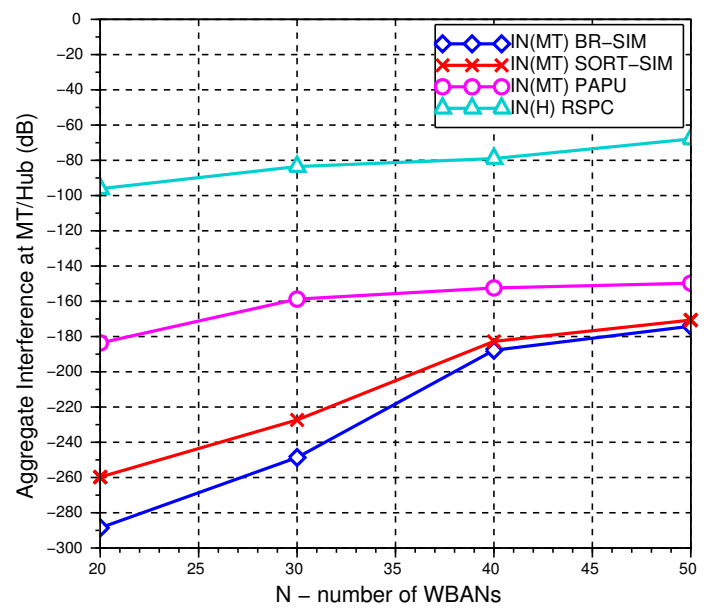

Figure 17: Aggregate interference at the Hub/MT.

component in the four algorithms, either with power control (PAPU and RSPC), or with constant transmit power (BR-SIM and SORT-SIM). Indeed, the four algorithms achieve efficient interference mitigation, ensuring feasible SIR values. However, the advantage of BR-SIM and SORT-SIM mainly appears when we compare the aggregate interference (Fig. 17) and the ZigBee SIR (Fig. 16b). This can be explained by the fact that our algorithms give some privilege to ZigBee links w.r.t. WiFi links; WiFi interference on ZigBee links is considered more crucial than ZigBee interference on WiFi links. In other words, our algorithms make sure that WiFi links (which use a transmit power 100 times higher than that of ZigBee) will not prevent ZigBee transmissions and deteriorate the BBN system performance.

Although the aggregate interference $I N(M T)$ of BR-SIM and SORT-SIM is significantly lower than that of RSPC $I N(H)$, it increases more rapidly for higher densities, because the use of orthogonal channels is no more possible, and BR-SIM and SORT-SIM start using channels with minimum mutual interference, with constant WiFi and ZigBee powers. However, RSPC main- 
tains approximately the same level of interference by adjusting the transmit power of the sensor/relay nodes. Hence, it would be interesting in future work to consider a control power mechanism together with the channel assignment to further improve the efficiency of the SIM game.

\section{Conclusion}

In this paper we studied the distributed interference mitigation problem in BBN scenarios from a game theoretical perspective. In particular, our work made three main contributions. First, we formulated the problem as a game considering the SIR, which accurately models the channel capacity that can be achieved in the presence of mutual and cross-technology interference. Second, we studied the properties of our game proving the existence of a Nash Equilibrium, which represents channel allocations that minimize the mutual and cross-technology interference. Third, we proposed a two-stage algorithm (called BR-SIM) based on the best-response dynamics to compute the Nash Equilibria in a distributed fashion. We further developed an alternative approach (SORTSIM) that reaches a sub-optimal solution in less computational time than BR-SIM. Finally, we evaluated and compared our SIM game theoretical approaches to (relay-assisted) power control schemes (i.e., PAPU and RSPC) in realistic BBN scenarios. We first showed that the BR-SIM algorithm converges quickly and achieves feasible values for the utility functions, while SORTSIM presents some practicability benefits under specific network scenarios. Then, we demonstrated that BR-SIM and SORT-SIM outperform PAPU and RSPC in terms of SIR and Aggregate Interference in several cases, and especially when the network density is quite low.

Besides, numerical results we gathered in the present work show that BBN scenarios require the definition of distributed scheduling algorithms to avoid simultaneous transmissions that might affect the channel quality and completely prevent communications among network nodes. 


\section{References}

[1] A. Meharouech, J. Elias, S. Paris, and A. Mehaoua. Socially-Aware Interference Mitigation Game in Body-to-Body Networks. In Proceedings of the International Conference on NETwork Games COntrol and oPtimization (NETGCOOP) 2014 (short paper), Trento, Italy, October 29-31, 2014.

[2] S.L. Cotton and W.G. Scanlon. Using smart people to form future mobile wireless networks. Microwave Journal, 54(12):24-40, 2011.

[3] M. Chen, S. Gonzalez, A. Vasilakos, H. Cao, and V.C.M. Leung. Body area networks: a survey. Mobile Networks and Applications, 16(2):171-193, April 2011.

[4] Simon L Cotton, William G Scanlon, and Bhopinder K Madahar. Millimeter-wave soldier-to-soldier communications for covert battlefield operations. Communications Magazine, IEEE, 47(10):72-81, 2009.

[5] Carles Gomez, Joaquim Oller, and Josep Paradells. Overview and evaluation of bluetooth low energy: An emerging low-power wireless technology. Sensors, 12(9):11734-11753, 2012.

[6] Carla F Chiasserini and Ramesh R Rao. Coexistence mechanisms for interference mitigation between ieee 802.11 wlans and bluetooth. In INFOCOM 2002. Twenty-First Annual Joint Conference of the IEEE Computer and Communications Societies. Proceedings. IEEE, volume 2, pages 590598. IEEE, 2002.

[7] IEEE Standards Association et al. Ieee standard for local and metropolitan area networks-part 15.6: wireless body area networks. IEEE Std, 802(6):2012, 2012.

[8] G. Fang, E. Dutkiewicz, K. Yu, R. Vesilo, and Y. Yu. Distributed inter-network interference coordination for wireless body area networks. In IEEE GLOBECOM'10, pages 1-5, Miami, Florida, USA, Dec. 2010.

[9] H.J. Lee, H. Kwon, A. Motskin, and L. Guibas. Interference-aware mac protocol for wireless networks by a game-theoretic approach. In IEEE INFOCOM'09, pages 1854-1862, Rio de Janeiro, Brazil, April 2009

[10] J. Chen, Q. Yu, P. Cheng, Y. Sun, Y. Fan, and X. Shen. Game theoretical approach for channel allocation in wireless sensor and actuator networks. IEEE Transactions on Automatic Control, 56(10):2332-2344, 2011.

[11] W.-B. Yang and K. Sayrafian-Pour. Interference mitigation for body area networks. In 22nd IEEE PIMRC'11, pages 2193-2197, Toronto, Canada, September 2011.

[12] S.L. Cotton, A. McKernan, A.J. Ali, and W.G. Scanlon. An experimental study on the impact of human body shadowing in off-body communications channels at 2.45 ghz. In Proceedings of the 5th IEEE European Conference on Antennas and Propagation (EUCAP), pages 3133-3137, Rome, Italy, April 2011.

[13] J. Huang, G. Xing, G. Zhou, and R. Zhou. Beyond co-existence: Exploiting wifi white space for zigbee performance assurance. In 18th IEEE International Conference on Network Protocols (ICNP'10), pages 305-314, Kyoto, Japan, 2010.

[14] S.Y. Shin, H.S. Park, S. Choi, and W.H. Kwon. Packet error rate analysis of zigbee under wlan and bluetooth interferences. IEEE Transactions on Wireless Communications, 6(8):2825-2830, 2007. 
[15] C.-J.M. Liang, N.B. Priyantha, J. Liu, and A. Terzis. Surviving wi-fi interference in low power zigbee networks. In Proceedings of the 8th ACM Conference on Embedded Networked Sensor Systems (SenSys'10), pages 309-322, Zurich, Switzerland, November 2010.

[16] J. Hou, B. Chang, D.-K. Cho, and M. Gerla. Minimizing 802.11 interference on zigbee medical sensors. In Proceedings of the Fourth International Conference on Body Area Networks (BodyNets'09), page 5. ICST (Institute for Computer Sciences, Social-Informatics and Telecommunications Engineering), Brussels, Belgium, 2009.

[17] Pedro BF Duarte, Zubair Md Fadlullah, Athanasios V Vasilakos, and Nei Kato. On the partially overlapped channel assignment on wireless mesh network backbone: A game theoretic approach. Selected Areas in Communications, IEEE Journal on, 30(1):119-127, 2012.

[18] Ramtin Kazemi, Rein Vesilo, and Eryk Dutkiewicz. A novel genetic-fuzzy power controller with feedback for interference mitigation in wireless body area networks. In Vehicular Technology Conference (VTC Spring), 2011 IEEE 73rd, pages 1-5. IEEE, 2011.

[19] S. Paris, J. Elias, and A. Mehaoua. Cross technology interference mitigation in body-to-body area networks. In IEEE WoWMoM'13, pages 1-9, Madrid, Spain, June 2013.

[20] A.H. Mohsenian Rad and V.W.S. Wong. Partially overlapped channel assignment for multi-channel wireless mesh networks. In IEEE ICC'07, pages 3770-3775, Glasgow, Scotland, June 2007.

[21] Vincent WS Wong et al. Joint optimal channel assignment and congestion control for multi-channel wireless mesh networks. In Communications, 2006. ICC'06. IEEE International Conference on, volume 5, pages 1984-1989. IEEE, 2006.

[22] Arunesh Mishra, Vivek Shrivastava, Suman Banerjee, and William Arbaugh. Partially overlapped channels not considered harmful. In ACM SIGMETRICS Performance Evaluation Review, volume 34, pages 63-74. ACM, 2006.

[23] R. Rosini, R. D'Errico, and R. Verdone. Body-to-body communications: A measurement-based channel model at $2.45 \mathrm{ghz}$. In IEEE PIMRC'12, pages 1763-1768, Sydney, Australia, Sept. 2012.

[24] Anand Prabhu Subramanian, Himanshu Gupta, Samir R Das, and Jing Cao. Minimum interference channel assignment in multiradio wireless mesh networks. Mobile Computing, IEEE Transactions on, 7(12):1459-1473, 2008.

[25] Monty Beuster, Michael Beigl, Daniel Rohr, Till Riedel, Christian Decker, and Martin Berchtold. Matrix routing-an interference range insensitive routing protocol for wireless sensor networks. In Applications and the Internet, 2008. SAINT 2008. International Symposium on, pages 137-140. IEEE, 2008.

[26] Kaixin Xu, Mario Gerla, and Sang Bae. Effectiveness of rts/cts handshake in ieee 802.11 based ad hoc networks. Ad Hoc Networks, 1(1):107-123, 2003.

[27] Jocelyne Elias, Stefano Paris, and Marwan Krunz. Cross technology interference mitigation in body area networks: an optimization approach. IEEE Transactions on Vehicular Technology, September 2014.

[28] S. Govindasamy, D.W. Bliss, and D.H. Staelin. Asymptotic Spectral Efficiency of Multiantenna Links in Wireless Networks With Limited Tx CSI. IEEE Transactions on Information Theory, 
58(8):5375-5387, 2012

[29] N. Golmie, D. Cypher, and O. Rebala. Performance analysis of low rate wireless technologies for medical applications. Elsevier Computer Communications, 28(10):1266-1275, 2005.

[30] N. Nisan, T. Roughgarden, E. Tardos, and V. V Vazirani. Algorithmic game theory. Cambridge University Press, 2007.

[31] X. Chen and J. Huang. Game theoretic analysis of distributed spectrum sharing with database. Proceedings of the IEEE 32nd International Conference on Distributed Computing Systems (ICDCS), pages 255-264, Macau, China, June 18-21, 2012.

[32] Zhaoyang Zhang, Honggang Wang, Chonggang Wang, and Hua Fang. Interference mitigation for cyber-physical wireless body area network system using social networks. Emerging Topics in Computing, IEEE Transactions on, 1(1):121-132, 2013.

[33] D. Monderer and L.S. Shapley. Potential games. Games and economic behavior, Elsevier, 14(1):124-143, 1996.

[34] INRIA. Scilab software package.

[35] Jie Dong and David Smith. Joint relay selection and transmit power control for wireless body area networks coexistence. In IEEE International Conference on Communications (ICC), pages 5676-5681. IEEE, Sydney, Australia, June 2014.

[36] David B Johnson and David A Maltz. Dynamic source routing in ad hoc wireless networks. In Mobile computing, pages 153-181. Springer, 1996.

[37] Jie Dong and David Smith. Opportunistic relaying in wireless body area networks: Coexistence performance. In IEEE International Conference on Communications (ICC), pages 5613-5618. IEEE, Budapest, Hungary, June 2013.

[38] D Smith, L Hanlen, D Rodda, B Gilbert, J Dong, and V Chaganti. Body area network radio channel measurement set. URL: http://www. opennicta. com/datasets. accessed December, 2012. 